\title{
The Effects of Visual Stimulation and Memory on Neurons of the Hippocampal Formation and the Neighboring Parahippocampal Gyrus and Inferior Temporal Cortex of the Primate
}

\author{
I. P. Riches, F. A. W. Wilson, ${ }^{a}$ and M. W. Brown \\ Department of Anatomy, University of Bristol, School of Medical Sciences, Bristol, BS8 1TD, United Kingdom
}

The activity of 736 single neurons was recorded from the hippocampal formation (HF), the rhinal cortex (RH), the medial and anterior inferior temporal cortex (TE), or areas TF and TH of the parahippocampal gyrus (PHG) of monkeys during the performance of a delayed matching to sample task. The results indicate differences between the areas in their contributions to sensory processing and memory. Of the neurons, $55 \%$ responded to either the first (S1) and/or the second (S2) of the two successively presented visual stimuli. The proportion of responsive neurons and the proportion of neurons that responded selectively on the basis of shape or color (but not size) were significantly higher in areas TE + RH than in HF+PHG. The responses to S1 differed from those to $\$ 2$ for $18 \%$ of the total sample: of these differentially responsive neurons, $66 \%$ of the TE $+\mathrm{RH}$ neurons responded more strongly to $S 1$ (the sample presentation, allowing stimulus acquisition), whereas $71 \%$ of the HF+PHG neurons responded more strongly to $\$ 2$ (the match/nonmatch comparison, when the behavioral decision could be made).

Of 239 TE+RH neurons recorded during the delayed matching task or when objects were shown, $12 \%$ displayed evidence of memory for the previous occurrence of stimuli by responding strongly to the first, but significantly less strongly to subsequent presentations of visual stimuli that were novel or had not been seen recently. In contrast, none $(0 \%)$ of 328 neurons so tested in HF and PHG had a response that declined significantly on stimulus repetition. For six $(86 \%)$ of seven TE +RH neurons tested, the decrement in response persisted even after distraction by intervening presentations of other stimuli. Further evidence of information storage was found for $7(33 \%)$ of 21 neurons for which responses to the first presentations of unfamiliar objects were significantly greater than to the first presentations of very familiar objects, even though the familiar objects had not been seen for $>15 \mathrm{~min}$.

Received July 18, 1990; revised Nov. 29, 1990; accepted Jan. 11, 1991.

We are grateful to Dr. S. P. Evans for statistical advice, J. A. Leendertz and C. M. de la Mahotiere for computer programming, R. Chambers, A. M. Somerset, and J. Long for technical assistance, and the Nuffield Foundation, Medical Research Council of the United Kingdom, and Wellcome Trust for financial support.

Correspondence should be addressed to Dr. M. W. Brown, Department of Anatomy, University of Bristol, School of Medical Sciences, University Walk, Bristol, BS8 1TD, UK.

a Present address: Fraser Wilson, Section of Neurobiology, Yale University, School of Medicine, 333 Cedar Street, New Haven, CT 06510.

Copyright (C) 1991 Society for Neuroscience $0270-6474 / 91 / 111763-17 \$ 03.00 / 0$
A considerable body of data from both animal and human studies has implicated the medial temporal lobes in memory processes (for reviews, see Mishkin, 1982; Parkin, 1987; Squire, 1987; Mayes, 1988). Of the structures in this region, most research has concerned the role of the hippocampal formation (HF): hippocampus, dentate gyrus (DG), and subicular cortex (SUB). However, in cases of memory impairment following lesions of the medial temporal lobes in either humans or monkeys, damage has commonly extended to the mcdial temporal cortex adjacent to the HF (e.g., Scoville and Milner, 1957; Horel, 1978; Mishkin, 1978). Thus, there is still uncertainty concerning the individual contributions to memory of the HF and of the neighboring medial temporal cortex. Even in cases where damage is restricted (e.g., Zola-Morgan et al., 1986), a lesion in one part of the medial temporal region may disrupt function in its other parts because the different parts of the region are so strongly interconnected.

The inferior temporal cortex projects into the HF via the cortex adjacent to the rhinal sulcus, that is, rhinal cortex: peri-, pro-, and entorhinal cortex (RH; Jones and Powell, 1970; Van Hoesen and Pandya, 1975; Rosene and Van Hoesen, 1987). Electrophysiological studies have shown that lateral inferior temporal cortical (LT) regions are important for the processing of visual information (e.g., Gross et al., 1972; Desimone and Gross, 1979; Fuster and Jervey, 1982; Baylis et al., 1987). The more medial and anterior inferior temporal and rhinal cortical regions have been little studied by such techniques. The same is true of areas TF and TH of the parahippocampal gyrus (PHG), though they also provide input to the rhinal cortex and form an essential part of one of the major output pathways of the HF (Rosene and Van Hoesen, 1977, 1987; Van Hoesen, 1982).

In the present experiments, neuronal responses were recorded in the HF and in the neighboring PHG and inferior temporal cortex during performance of a delayed matching task by monkeys. Damage to the medial temporal lobes has been reported as impairing performance of delayed matching tasks by humans or monkeys (Stepien et al., 1960; Correll and Scoville, 1965; Sidman et al., 1968; Mishkin, 1978; Mahut et al., 1982; Malamut et al., 1984; Zola-Morgan and Squire, 1985; Squire et al., 1988); cooling or lesioning of the anterior inferior temporal gyrus also causes a similar deficit in monkeys (Horel et al., 1987). Further, a previous study (Brown, 1982) indicated that hippocampal neurons were responsive during monkeys' performance of such a task. The aim of the present study was to compare neuronal responses recorded using the same techniques in the same experiments between the various areas, in order to look for differences in the contributions of parts of the medial tem- 
poral lobes to visual and mnemonic processing. For example, it was hypothesized that, compared to responses in the inferior temporal cortex, neuronal responses in the HF might be influenced more by factors other than the physical characteristics of the sensory stimuli. The results presented here provide evidence in support of this hypothesis. Moreover, if the medial temporal lobe is important for recognition memory (Mishkin, 1982; Parkin, 1987; Squire, 1987; Mayes, 1988), then differences between responses to familiar and unfamiliar stimuli might be expected. Hence, as well as recording responses to familiar stimuli presented during the delayed matching task, it was decided to investigate whether there were changes in the responses when new, unfamiliar stimuli were introduced into the task. Such changes were indeed found; they were further studied outside the behavioral task by showing the animals objects of varying familiarity.

Some preliminary findings have been previously published (Brown et al., 1987).

\section{Materials and Methods}

Subjects and behavioral task. Monkeys (three Macaca mulatta and one Macaca fascicularis) weighing between 3 and $8 \mathrm{~kg}$ were trained to perform a Konorski (Konorski, 1959) delayed matching to sample task prior to making recordings of the activity of single neurons. In this task (see Fig. 1), the cue for the start of a trial was diffuse illumination of a monitor screen for $0.5 \mathrm{sec}$ by colored light. After an interval of $0.5 \mathrm{sec}$, a visual stimulus ( $\mathrm{S} 1$ ) was shown on the screen for $0.5 \mathrm{sec}$, followed, after another $0.5-\mathrm{sec}$ interval, by a second visual stimulus (S2). S2 was also shown for $0.5 \mathrm{sec}$. The monkey was rewarded with fruit juice $(\approx 0.5$ $\mathrm{ml}$ ) for a press to a right-hand panel made $0.5-5.0 \mathrm{sec}$ after the offset of $\mathbf{S} 2$, if $\mathrm{S} 1$ and $\mathbf{S} 2$ were the same. A press to a left-hand panel during the same interval was rewarded if S1 and S2 had been different. Other presses resulted in the lights being dimmed for a few seconds in the light-proof, sound-attenuating cubicle within which the monkey was trained. The intertrial interval was variable $(2-5 \mathrm{sec})$. Training was initially to a left/right visual discrimination (constant vs. flashing stimulus), followed by successive approximation to the delayed matching task.

During training and recording sessions, the monkey was seated in a primate chair facing a 22-cm-square Perspex panel divided vertically into three equal sections. The middle section was fixed; the right and left sections were hinged at the top and were spring loaded against microswitches that recorded the animal's presses. Stimuli were displayed centrally on a color (RGB) videomonitor immediately behind the Perspex, approximately $30 \mathrm{~cm}$ from the monkey's eyes. The stimuli subtended between $2^{\circ}$ and $10^{\circ}$ and were of a variety of different geometric shapes, a pool of $>80$ being available. Each stimulus was one of seven possible colors, though only a subset of the 560 total possible color and shape combinations were used in the experiments. A few stimuli were used frequently, the most commonly used being a white square; other stimuli were relatively unfamiliar, having been seen only on rare occasions if at all by the monkey. On trials when $S 1$ and $S 2$ differed, the difference was most commonly of size, with shape and color being held constant. The trial types were controlled by computer to give a balanced, pseudorandom sequence. The four possible trial types (see Fig. 1) were constructed using a single pair of stimuli (e.g., a large and a small white square) for a block of 8-20 trials before being changed. The monkey's hand and eye movements were videomonitored and recorded. Trials during which the monkey failed to fixate on the monitor screen were excluded from subsequent analysis. Trials on which the animal made errors were analyzed separately; such trials were comparatively few because the monkeys usually performed at levels of $>95 \%$ correct, being highly practiced at the task.

During recording sessions with two monkeys, the delayed matching task was interrupted from time to time, and the Perspex screen and videomonitor were removed; this allowed stimuli to be shown to the monkey for a few seconds through the resultant hole and against a white background. The stimuli were drawn from a collection of $>1000$ small objects varying greatly in shape, color, and size. Some were very familiar to the animal, while others it had never, or only rarely, seen before. Stimuli also included pieces of fruit and a syringe containing fruit juice; the monkey was allowed to eat the fruit and drink the juice. Presentations of stimuli and food were made in a pseudorandom order so that the monkey could not predict when food would be shown or when a particular stimulus would be repeated.

Neuronal recording. When a monkey could regularly perform the delayed matching task at $>90 \%$ correct, it was prepared under anesthesia using aseptic techniques for the recording of single neurons by fixing a chamber made of titanium to the skull. Two weeks were allowed for recovery from the operation, after which recordings were made using movable tungsten microclcctrodes (Horn, 1969). Potentials of single, well-isolated neurons were conventionally amplified, monitored, and displayed before being recorded on an FM tape recorder and a polygraph. A pulse-shape discriminator (Brown and Leendertz, 1979) enabled potentials from simultaneously recorded neurons to be processed separately by computer (Acorn Computers, model B, BBC). The computer stored data (times of action potentials with respect to trial events, to the nearest millisecond) for later processing by a mainframe computer and also allowed raster displays and peristimulus histograms to be generated on line.

Data analysis. Spike counts in successive 0.5 -sec intervals were used for data analysis. For the delayed matching task, all reported neurons were recorded for $>25$ correct trials. The significance of neuronal responses was determined by comparing the activity in the $1 \mathrm{sec}$ before the start of a trial (i.c., prior to the onset of the cuc) with the activity in the $1 \mathrm{sec}$ following the onset of a stimulus by means of paired $t$ tests, to establish the consistency of changes in firing rate across repeated trials. No neurons have been excluded because of their lack of responsiveness. The reliability of differences in response to different types of stimuli was determined using multivariate analyses of variance (MANOVA). Where appropriate (small numbers of trials or low spike counts), logarithmic transformations of the spike counts were employed. When objects were shown, responses were determined from spike counts taken during the $2 \mathrm{sec}$ following stimulus onset.

Because for any given analysis neurons were categorized as either responsive or not, the underlying data distribution may be expected to be binomial. Hence, the proportions of responsive neurons in the various brain regions were subjected to a logit transformation and analyzed by an unbalanced two-way analysis of variance with brain area and monkey as factors, using a generalized linear model with binomial error distribution (Baker and Nelder, 1978). The variation (deviance) associated with the main effects of the two factors "brain area" and "monkey" were isolated as $\chi^{2}$ values [identified in the text as binomial error model (BEM) $\chi^{2}$ values] by calculating the change in deviance when one of the factors was removed from a model including both. After account had been taken of the variation due to brain areas and monkeys, the residual variation was in every case less than that expected by chance, indicating the adequacy of the model used. This analysis of variance allowed the significance of differences between the regions to be established in the presence of variation between monkeys. All statistical tests used a significance level of 0.05 and were two tailed unless otherwise stated.

Histological localization. The depth of cach neuron was noted at the time of recording. Their positions were plotted on large-scale drawings (taken at $1-\mathrm{mm}$ intervals) from cresyl violet-stained, $50-\mu \mathrm{m}$-thick serial sections of the animals' temporal lobes. These positions were determined by making measurements from lateral and anterior-posterior $\mathrm{x}$-ray photographs of the microelectrodes $i n$ situ, the electrode's position in each case being compared to that of fixed reference electrodes and skull landmarks, and from the location of microlesions made through the microelectrode where responsive neurons were found (corrections for $x$-ray expansion and brain shrinkage were made). The boundaries between the various regions were determined from the serial sections using published criteria (Lorente de Nó, 1933, 1934; von Bonin and Bailey, 1947; Van Hoesen and Pandya, 1975; Seltzer and Pandya, 1978; Cassell and Brown, 1984). The boundary of perirhinal cortex with the medial and anterior inferior temporal cortex follows Van Hocsen and Pandya (1975); that of Zola-Morgan et al. (1989) would place some of the medial part of area TE (TE1) as used in this paper within perirhinal cortex.

\section{Results}

This section is divided into those results obtained during the delayed matching task and those obtained when the monkeys were shown objects. Analyses of responses to familiar stimuli 


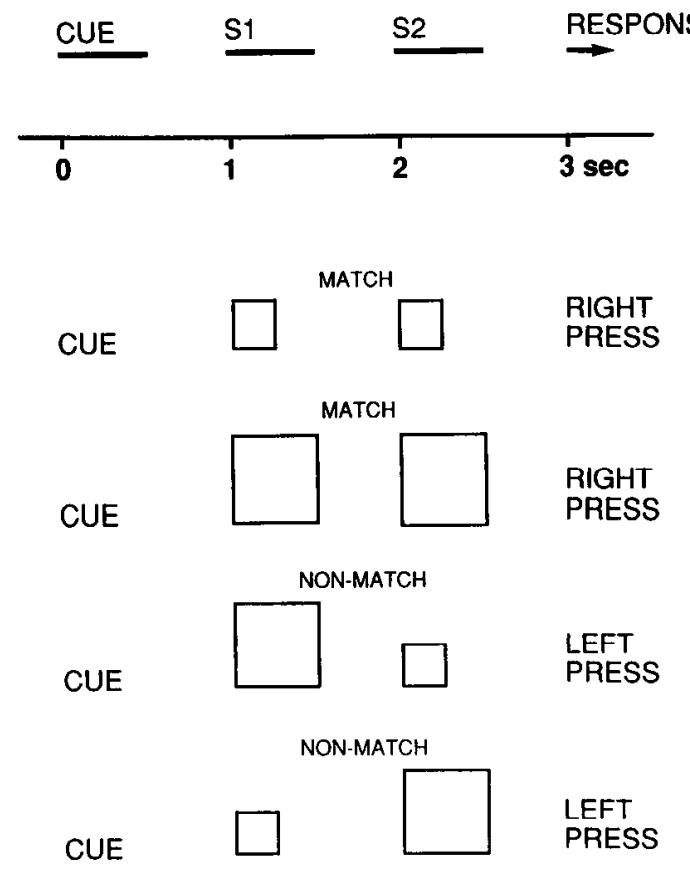

Figure 1. The timings of events during the delayed matching task. The task consisted of a cue followed by two successively presented stimuli (S1 and S2). If S1 and S2 were the same (match), a right (panel) press was correct; if they differed (nonmatch), a left press was correct. To be correct, presses also had to be made between 0.5 and $5.0 \mathrm{sec}$ after the offset of $\mathbf{S 2}$. The four possible trial types are illustrated for a stimulus pair consisting of a large and a small square.

are followed by analyses of changes in response to changes in stimulus familiarity.

\section{Responses in the delayed matching task}

A total of 736 single neurons were recorded from the hippocampal region and adjacent temporal cortex during performance of the delayed matching task. Task details are given in Figure 1. The distribution of the neurons in the various anatomical rcgions and a summary of the abbreviations used for the eight areas into which they have been grouped are given in Table 1. The number of animals was not sufficiently large to establish any consistent differences between data obtained from the right or left hemispheres, even taking account of the preferred hand (monkeys variously used left, right, or left and right hands to press the panels). The quoted figures have been averaged across hemispheres and monkeys, though the statistical tests take account of between-animal variation. There were no statistically significant interactions between the factors "monkey" and "brain area."

\section{Responses to the cue}

Of the total sample, $17.1 \%$ (126 of 736 neurons) responded to the cue that initiated a trial: $13.7 \%$ with an increase in activity and $3.4 \%$ with a decrease. The proportions of responsive neurons in the various areas are shown in Figure $2 a$. These proportions werc subjected to an ANOVA using a binomial error distribution. The proportions of neurons showing significant increases in activity varied significantly $\left(\mathrm{BEM} \chi^{2}=44.93 ; \mathrm{df}=\right.$ $7 ; P<0.001$ ) between the various areas; there were no such reliable differences for the proportions of neurons showing significant decreases in activity. Further analysis revealed that the variation between the areas arose because the proportion $(30.2 \%)$ of neurons showing increased activity to the cue in areas TE1 and $\mathrm{RH}$ combined was significantly higher than that $(10.2 \%)$ in the remaining areas. No other differences between the areas reached significance, and there was no significant interaction between the factors "brain area" and "monkey."

\section{Mean responses to $S 1$ and $S 2$}

Of the 736 neurons, $406(55.2 \%)$ responded to $S 1$ and/or S2 of the successively presented visual stimuli. The proportions of ncurons that responded to $S 1$, to $S 2$, or to both $S 1$ and $S 2$ for the different areas are shown in Figure $2 b$. The proportions of responsive neurons varied between the different areas. Analysis revealed that both for $\mathrm{S} 1$ and for $\mathrm{S} 2$ this variation is explained by the significantly higher proportion of responsive neurons in areas LT, TE1, and RH combined (TE+RH neurons) than in the remaining areas (HF+PHG neurons; details are given in Fig. 2 caption). The same pattern of significant variation between the areas emerged when only neurons that significantly increased their activity to $\mathrm{S} 1$ or $\mathrm{S} 2$ were considered (see Fig. $2 c$ ). The incidence of neurons whose activity decreased did not vary significantly between the areas. Thus, overall, a higher propor-

Table 1. The numbers of neurons recorded in the various regions during the performance of the delayed matching task, the number that were responsive, and the median, minimum, and maximum background neuronal firing rates $^{a}$

\begin{tabular}{lcccccccc} 
& LT & TE1 & RH & DG & CA3 & CA1 & SUB & TF + TH \\
\hline Total recorded & 27 & 78 & 24 & 121 & 158 & 92 & 133 & 103 \\
Responsive & 17 & 50 & 17 & 61 & 91 & 55 & 61 & 54 \\
Median SA & 2.6 & 4.3 & 5.2 & 6.5 & 7.2 & 5.7 & 5.3 & 4.2 \\
Minimum SA & 0.5 & 0.5 & 0.7 & 0.1 & 0.1 & 0.2 & 0.3 & 0.1 \\
Maximum SA & 10.2 & 15.2 & 14.1 & 48.2 & 54.5 & 42.4 & 31.9 & 25.2
\end{tabular}

Area definitions: LT, lateral inferior temporal cortex; TE1, medial part of area TE; RII, pro-, peri-, and entorhinal cortex; DG, dentate gyrus (including its hilus); CA3 and CA1, subfields of hippocampus; SUB, subiculum plus pre-, pro-, and parasubiculum; TF + TH, areas TF and TH of the parahippocampal gyrus. Note that LT includes all of area TE except TE1 (Scltzcr and Pandya, 1978) and that the boundary of TE with RH follows the more medial position of Van Hoesen and Pandya (1975) rather than that of Zola-Morgan et al. (1989). No clear differences were noted between neurons recorded in anatomical subdivisions of these eight areas, though it should be noted that in such subregions the sample sizes were not large. SA, spontaneous activity.

${ }^{a}$ The background firing rate for each neuron was defined as the mean activity occurring during the intertrial intervals of the task. 
a

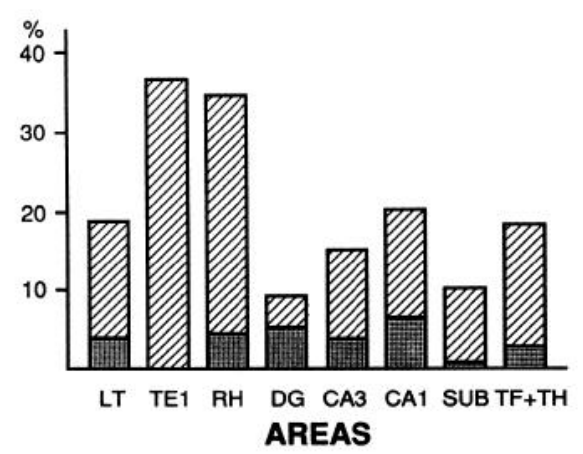

Increases in
firing rate to cue
Decreases in
firing rate to cue b

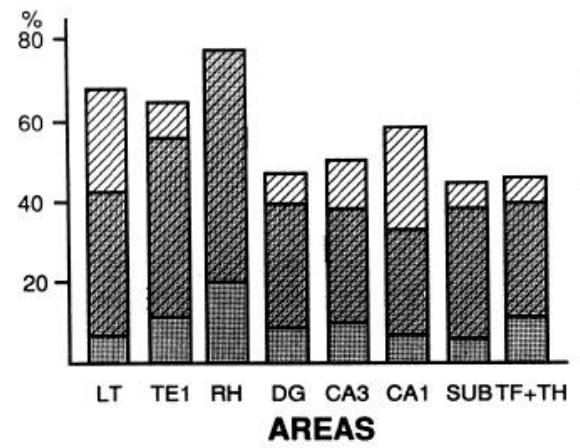

Neurons responsive
to S2
$\begin{aligned} & \text { Neurons responsive } \\ & \text { to S1 }\end{aligned}$
$\begin{aligned} & \text { Area of overlap: } \\ & \text { neurons responsive } \\ & \text { to both } \mathrm{S} 1 \text { and } \mathrm{S} 2\end{aligned}$
C

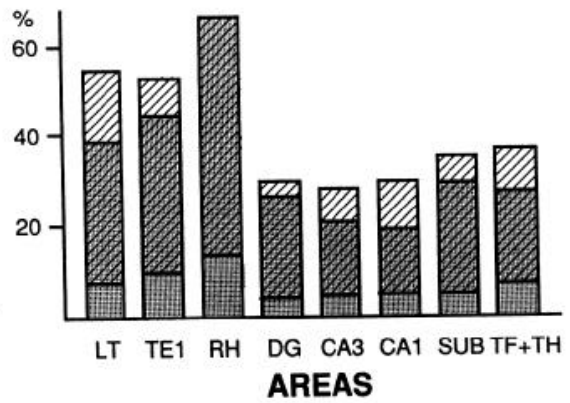

Increases in activity
to S2
Increases in activity
to S1
Area of overlap:
increases in activity
to S1and S2

Figure 2. Proportions (\%) of neurons responding in the delayed matching task for the eight areas sampled. The areas are summarized in Table 1. $a$, Proportions for response to the cue. $b$, Proportions for response to S1 (shaded part of bar) or S2 (hatched part of bar); the part of the histogram with shading overlapping hatching represents the proportions responsive to $\mathrm{S} 1$ and $\mathrm{S} 2$; the full height of the histogram represents the total proportion responsive (i.e.,to S1 and/or S2). Analysis revealed that both for S1 and for S2 there was a significantly (BEM $\chi^{2}=24.1$ and 18.7 , respectively; df $=7 ; P<0.01$ in both cases) higher proportion of responsive TE $+\mathrm{RH}$ neurons than HF $+\mathrm{PHG}$ neurons; $56.6 \%$ of TE $+\mathrm{RH}$ neurons responded to $\mathrm{S} 1$, and $57.4 \%$ to $\mathrm{S} 2$, whereas only $39.9 \%$ of $\mathrm{HF}+\mathrm{PHG}$ neurons responded to $\mathrm{S} 1$, and $44.5 \%$ to $\mathrm{S} 2$. No other differences between the areas reached significance. Additionally, the proportion of TE + RH neurons that responded to both S1 and S2 was significantly higher than that for HF+PHG (48.8\%:31.3\%). No other differences reached significance. Because the task employed a constant interstimulus interval between S1 and S2, it is possible that for some neurons prolonged or delayed activity changes as a result of the presentation of S1 could have contributed to changes seen during the presentation of S2. However, this possibility is not high because there was little evidence of strong responses continuing more than 0.5 $\mathrm{sec}$ after the offset of S2, nor was there any evidence for such responses to the sight of objects presented outside the task. $c$, Proportions for increase in activity to $\mathrm{S} 1$ or $\mathrm{S} 2$ (indicated as in $b$ ). Analysis (BEM $\chi^{2}$ for $\mathrm{S} 1$ and for $\mathrm{S} 2=24.9$ and 15.0 , respectively; $\mathrm{df}=7 ; P<0.001$ and $\mathrm{P}<0.02$, respectively) indicated that the proportion of responsive TE $+\mathrm{RH}$ neurons was nearly twice as high as that of $\mathrm{HF}+\mathrm{PHG}$ neurons (45.0:23.6\% for $\mathrm{S} 1 ; 43.4: 24.5 \%$ for $\mathrm{S} 2$ ). No other differences reached significance. Neither the proportion of neurons whose response was a decrease in activity to S1 (15.5\%) nor that for S2 (18.6\%) differed significantly between the various areas.

tion of TE+RH neurons $(65.1 \%)$ than of $\mathrm{HF}+\mathrm{PHG}$ neurons $(53.1 \%)$ responded to $\mathrm{S} 1$ and/or $\mathrm{S} 2$.

The magnitudes of the responses are illustrated in Figure 3. There is a significant difference (Kolmogorov-Smirnov test) between the distributions of $\mathrm{HF}+\mathrm{PHG}$ and of $\mathrm{TE}+\mathrm{RH}$ neurons by size of response because (consistent with the findings reported above) a higher proportion of the responses of TE $+\mathrm{RH}$ than of $\mathrm{HF}+\mathrm{PHG}$ neurons are increases in activity. However, when the mean of the modulus of the percentage change in response is calculated, this does not differ significantly (ANOVA) between the eight different areas; that is, responses were of similar sizes in the different areas.

\section{Differences in response to $S 1$ compared to $S 2$}

If the physical attributes of the stimuli were the only determinants of neuronal response, then for the same stimuli the response to S2 should be the same as that to S1. However, examples of neurons that responded more to S1 than S2 or vice versa may be seen in Figure 4. Indeed, there was a significant difference (paired $t$ tests) between the mean response to $\mathrm{S} 1$ and that to S2 for $134(18.2 \%)$ of the 736 recorded neurons; the location of such differentially responsive neurons is given in Figure 5. Figure $6 a$ shows the proportions of such neurons in the different areas. For 85 neurons $(11.5 \%$ of the total sample of neurons, $63.4 \%$ of the differential neurons), the response to $\mathrm{S} 2$ was greater than that to $\mathrm{S} 1$. There was no significant variation in the proportion of such neurons between the different areas, though the overall proportion of HF+PHG neurons (12.4\%) for which the response to $\mathrm{S} 2$ was greater than that to $\mathrm{S} 1$ was higher than that $(7.8 \%)$ for TE $+\mathrm{RH}$ neurons. However, there was a significant $\left(\mathrm{BEM} \chi^{2}=20.81 ; \mathrm{df}=7 ; P<0.01\right)$ difference between the areas in the proportion of neurons for which the response to S1 was greater than that to S2. Further analysis indicated that the difference was due to the proportion of such $\mathrm{HF}+\mathrm{PHG}$ neurons $(4.9 \%)$ being significantly lower than that (14.7\%) of TE $+\mathrm{RH}$ neurons. Thus, of the differentially responsive neurons, $71 \%$ of the HF+PHG neurons responded more strongly to $S 2$ than to $S 1$, whereas $66 \%$ of the TE+RH neurons responded more strongly to $S 1$ than to S2.

\section{Selective responses related to shape, color, and/or size of the visual stimuli}

The responses of certain neurons varied with changes in the physical characteristics (shape, color, and/or size) of the stimuli; variations extended from neurons that responded to all stimuli tested, though with differences in the magnitude of the responses to the different stimuli, to neurons that responded to only one of the various stimuli tried. In Figure $7 a$, an example of a neuron is shown that responded to red-colored stimuli but not to stimuli of other colors. Overall, 213 of 736 neurons (28.9\%) responded differentially (MANOVA) to different stimuli. The selectivity of neuronal responses was not exhaustively explored in these 
experiments. However, to provide some indication of whether the recorded neurons signaled particular physical attributes of the stimuli, responses to stimuli that were white squares were compared to those to stimuli that were not, and additionally, the effect of the size of the stimulus on the response was analyzed. These features were selected because the white-square stimuli were presented to all the neurons, and because the difference between $S 1$ and $S 2$ was most commonly one of size rather than of shape or color.

The neuron illustrated in Figure $7 b$ was responsive to a variety of stimuli, but not to white squares. Significant differences in response dependent upon whether the stimulus was a white square or not were found for 171 of $736(23.2 \%)$ of the neurons. The proportions differed significantly between the various areas for both $\mathrm{S} 1$ and $\mathrm{S} 2$ (see Fig. $6 b$ ). In each case, the difference was based on a higher proportion of differentially responsive $\mathrm{TE}+\mathrm{RH}$ than $\mathrm{HF}+\mathrm{PHG}$ neurons, so that overall more than twice as many $\mathrm{TE}+\mathrm{RH}$ as $\mathrm{HF}+\mathrm{PHG}$ neurons responded differently when the stimulus changed from being a white square (44.1\%:18.9\%).

The neuron illustrated in Figure $7 c$ responded when $\mathrm{S} 1$ was a small square, but not when $\mathrm{S} 1$ was a large square. Overall, 121 neurons $(16.4 \%)$ responded significantly differently to large compared to small stimuli (either squares or other shapes); the proportion of such neurons in the various areas are given in Figure $6 c$. Such changes were found for responses to $\mathrm{S} 1$ for $9.8 \%$ of the neurons and to $\mathrm{S} 2$ for $9.2 \%$ of them. These proportions did not vary significantly between the various areas.

\section{Neuronal responses during delays}

Of the 315 neurons responsive to $S 1$, for $85(27.0 \%)$ the change in activity was greater in the $0.5 \mathrm{sec}$ following (i.e., during the delay between S1 and S2) than during the 0.5 -sec presentation of $\mathrm{S} 1$. Of the 345 neurons responsive to $\mathrm{S} 2$, for $65(18.8 \%)$ the change in activity was greater in the $0.5 \mathrm{sec}$ following S2 (i.e., during the delay following S2 and before the panel press) than during the 0.5 -sec presentation of S2. There were no significant differences in the incidence of such responses between the various areas.

\section{Regional differences in hippocampal neuronal responsiveness}

The proportion of neurons responsive to $S 1$ and/or $S 2$ in the $\mathrm{HF}$ (CA1, CA3, and SUB) $>10 \mathrm{~mm}$ anterior to the interaural line was compared to that posterior to this level. [The division corresponds to that made by Watanabe and Niki (1985).] The proportion found posteriorly $(59.7 \% ; 176$ of 295$)$ was significantly (BEM $\chi^{2}=10.7 ; \mathrm{df}=1 ; P<0.01$ ) greater than that anteriorly $(44.0 \% ; 92$ of 209). The incidence of neurons that responded significantly differently to $\mathrm{S} 2$ than to $\mathrm{S} 1$ was also significantly (BEM $\chi^{2}=16.1$; df $\left.=1 ; P<0.001\right)$ higher in the posterior $(22.0 \% ; 65$ of 295$)$ than the anterior $(8.1 \% ; 17$ of 209$)$ HF. [The difference remained significant (BEM $\chi^{2}=9.6$; $\mathrm{df}=$ $1 ; P<0.01)$ even when differential responses were expressed as a proportion of responsive neurons.]

\section{Complex-spike and $\theta$ cells}

Although some neurons in CA3 and CA1 of the hippocampus fired bursts of action potentials (complex spikes), not all neurons could readily be categorized as complex-spike or $\theta$ cells on the basis of criteria established in subprimate species (Fox and Ranck, 1975). No obvious differences in responsiveness were found between classes of cells categorized on the basis of these criteria.
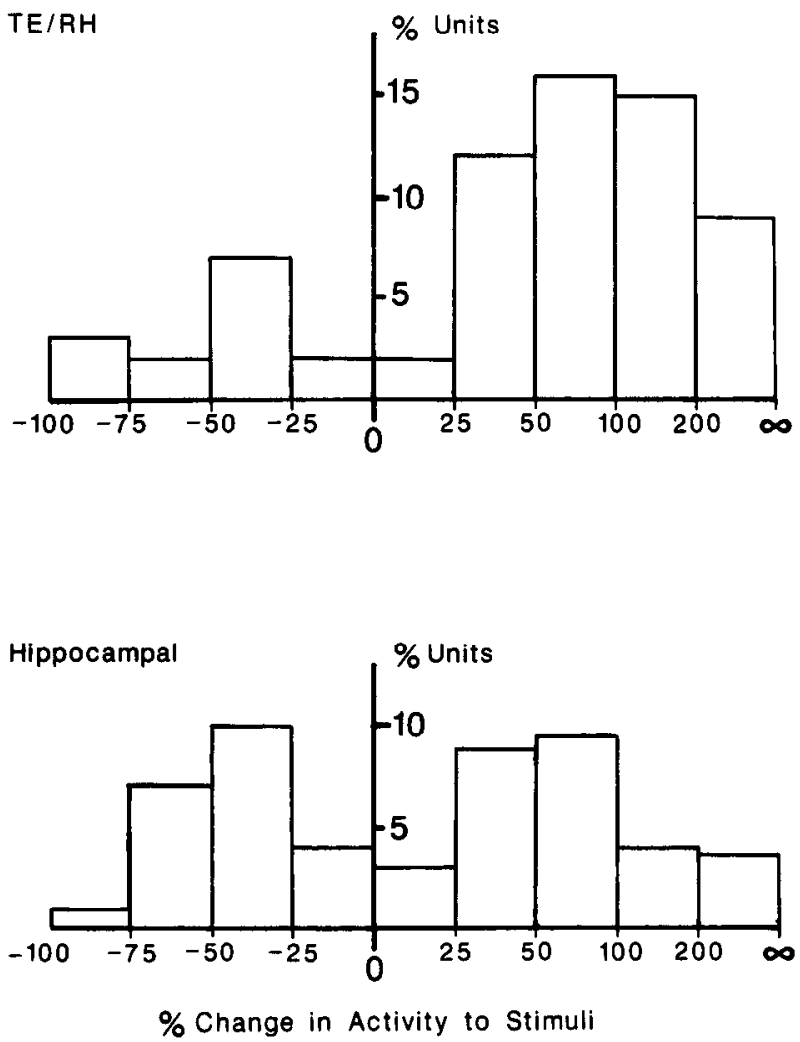

Figure 3. For TE+RH and hippocampal (i.e., HF+PHG) neurons, the proportions (percent of the total number of neurons recorded) having various magnitudes of response (the percent change from pretrial activity for $S 1$ or $S 2$, using whichever was the greater for each neuron). The proportions of neurons for which there was no significant response were not included. Note that there was a greater proportion of activity increases for TE $+\mathrm{RH}$ than for HF + PHG neurons, though the mean of the modulus of the response did not differ between the two regions.

\section{Pairs of neurons}

Simultaneous records of the activity of two neurons were obtained from 185 different locations. Both neurons were responsive to $\mathrm{S} 1$ and/or $\mathrm{S} 2$ for $72(38.9 \%)$ of these pairs; both in $\mathrm{HF}+\mathrm{PHG}$ and in TE $+\mathrm{RH}$, the proportions were slightly greater than would be expected by the chance pairing of responsive neurons, but not significantly so (binomial tests). Of the 185 pairs, in $15(8.1 \%)$ cases both neurons responded significantly differently to $S 1$ than to $S 2$. The incidence of such pairs in TE+RH (7 of $38 ; 18.4 \%$ ), but not those in HF+ PHG ( 8 of 147 ; $5.4 \%$ ), was significantly higher than would be expected by the chance pairing of such differentially responsive neurons (binomial test, $P<0.01$ ).

\section{Changes in response when unfamiliar stimuli were repeated}

The responses of certain TE $+\mathrm{RH}$ neurons were noted to be much stronger to the first than to subsequent presentations of visual stimuli that had not been seen recently. An example is shown in Figure $8 a$. Accordingly, responses in the delayed matching task to all stimuli that had not been seen recently were analyzed for evidence of response decrement with stimulus repetition. Thus, for each neuron the first occurrence (as S1) of each stimulus that had not been seen recently was compared to its next occurrence as $S 1$. In total the responses of $12(8.1 \%)$ of $148 \mathrm{TE}+\mathrm{RH}$ neurons could be shown (paired $t$ tests) to decline 


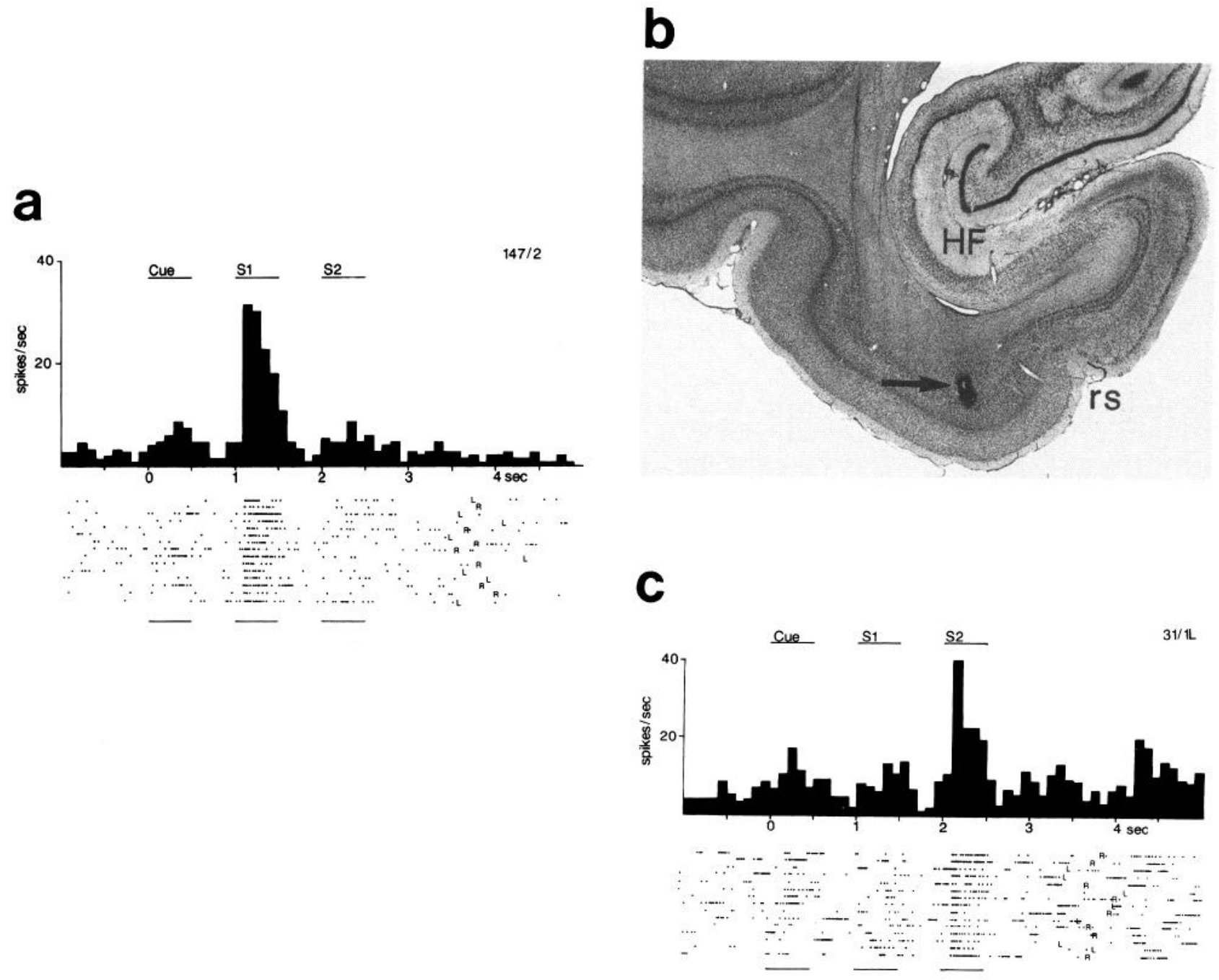

Figure 4. Differential responsiveness to S1 and S2. a, Histogram and rasters of activity of a neuron responding more strongly to S1 than to S2 or the cue. In the rasters, each line is a single trial, with dots representing the occurrence of action potentials. The time of the animal's left $(L)$ or right $(R)$ panel presses are indicated. Different stimuli were presented on the various trials illustrated, but there were no significant differences in activity between match or nonmatch trials, or between stimuli differing in size, shape, or color. $b$, Lesion (arrow) made in TE1 near its border with perirhinal cortex (Van Hoesen and Pandya, 1975) at the site where the neuron illustrated in $a$ was recorded. Note that this neuron would be located in perirhinal cortex by the criteria of Zola-Morgan et al. (1989). $H F$, hippocampal formation; $r s$, rhinal sulcus. $c$, Histogram and rasters of activity of a neuron recorded in the DG that responded more strongly to S2 than to S1 or the cue. Different stimuli were presented on the various trials illustrated, but there were no significant differences in activity between match or nonmatch trials, or between stimuli differing in size, shape, or color.

significantly upon stimulus repetition. There was a significant $\left(\mathrm{BEM} \chi^{2}=39.1 ; \mathrm{df}=7 ; P<0.001\right)$ difference in the incidence of these neurons between the different areas (Table 2). Further analysis revealed that the incidence of neurons with declining responses was highest in $\mathrm{RH}(8$ of $41 ; 19.5 \%)$, being significantly $(P<0.01)$ higher than in TE (TE1 +LT; 4 of $107 ; 3.7 \%)$, which in turn was significantly $(P<0.001)$ higher than in $\mathrm{HF}+\mathrm{PHG}$, where no such responses were found ( 0 of $292 ; 0 \%)$. The same statistical result was obtained if the number of responsive neurons in each area was used as the denominator. The locations of the neurons with declining responses are shown in Figure 9.

For one neuron, there were sufficient data to establish the significance (paired $t$ test) of the decrement in response that remained after intervening blocks of trials of other stimuli. For this neuron (in prorhinal cortex), the decrement in response persisted over intervals of $>60 \mathrm{sec}$ filled by other trials $(>10)$ of the delayed matching task.
In addition to the $12 \mathrm{TE}+\mathrm{RH}$ neurons whose responses were shown to decline significantly, there were other neurons for which the response had a tendency to decline on stimulus repetition, even though not significantly so. The response to the first presentations of stimuli as S1 was greater than the mean S1 response for $80 \%$ (43 of 54 ) of the TE+RH neurons that responded with an increase in activity to $\mathrm{S} 1$; this proportion is very significantly higher than the $50 \%$ expected by chance (binomial test, $P<0.0001$ ). No such significant difference was found for $\mathrm{HF}+\mathrm{PHG}$ neurons.

\section{Responses to objects}

\section{Mean responses}

Responses were also sought to the sight of objects for 271 neurons; 71 of 162 of these neurons in TE $+\mathrm{RH}$ and 73 of 109 in $\mathrm{HF}+\mathrm{PHG}$ were also recorded during the delayed matching task. The proportions of responsive neurons in the various areas are 


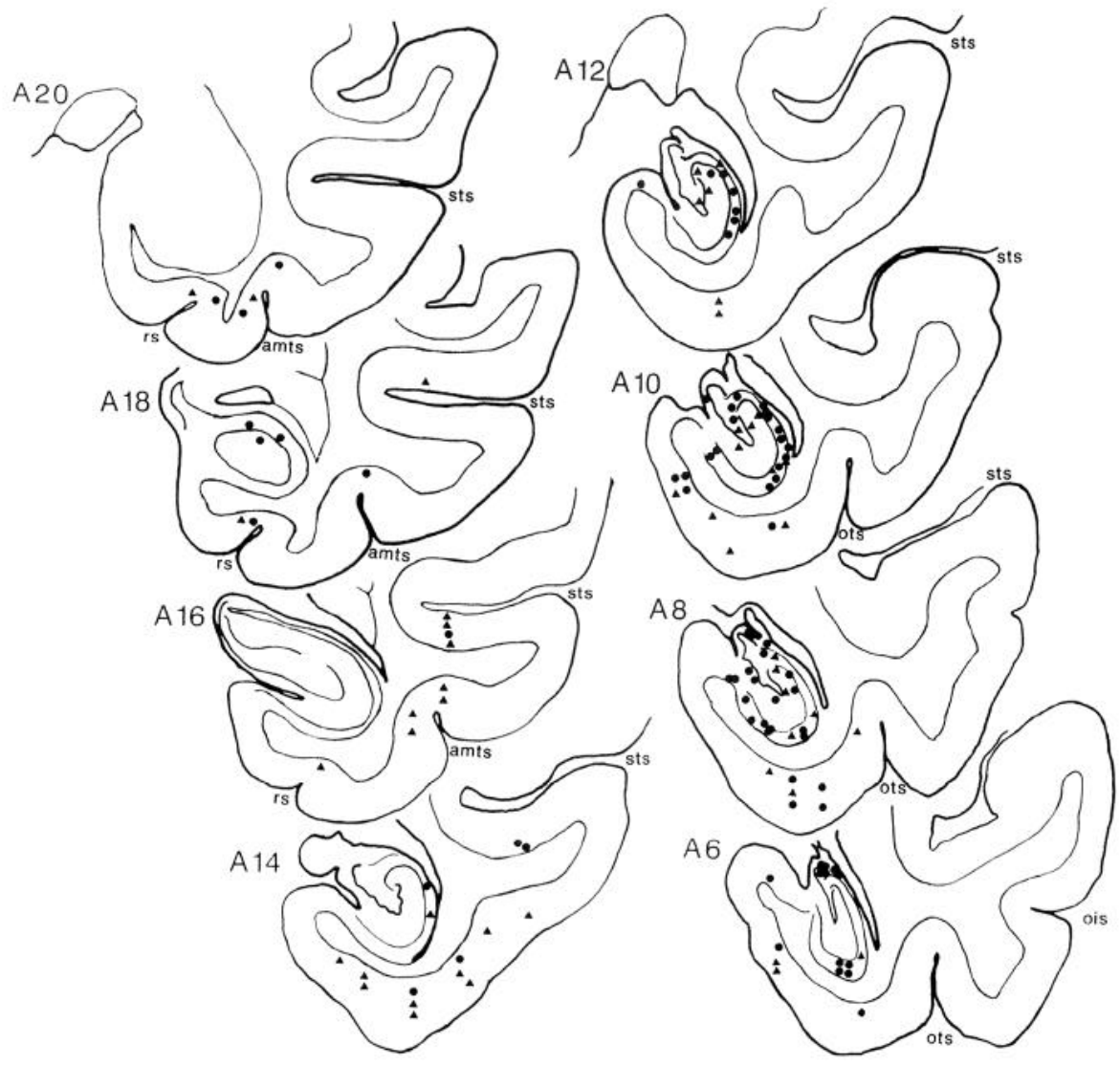

Figure 5. The locations of neurons for which the response to $\mathrm{S} 1$ was significantly greater (triangles) or smaller (circles) than that to $\mathrm{S} 2$. The locations are plotted onto reconstructions of sections through the medial temporal lobes every $2 \mathrm{~mm}$ from 6 to $20 \mathrm{~mm}$ anterior $(A)$ to the interaural line. No distinction has been made between neurons recorded in the left or right hemispheres. amts, anterior medial temporal sulcus; ois, occipito-inferior sulcus; ots, occipitotemporal sulcus; $r s$, rhinal sulcus; $s t s$, superior temporal sulcus. a

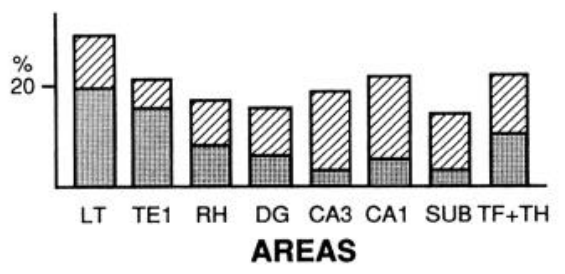

Response to $\mathrm{S} 2>$ response to $\mathrm{S} 1$

Response to $\mathrm{S} 1>$ response to S2 b

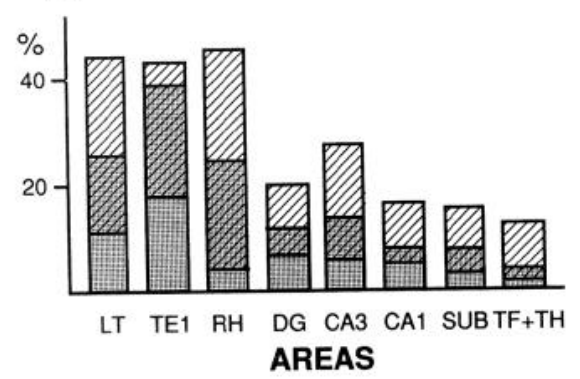

Responses to square differ from
responses to non-square (S2)
$\begin{aligned} & \text { Responses to square differ from } \\ & \text { responses to non-square (S1) }\end{aligned}$
$\begin{aligned} & \text { Area of overlap: } \\ & \text { responses to square differ from } \\ & \text { responses to non-square (S1 and S2) }\end{aligned}$
C

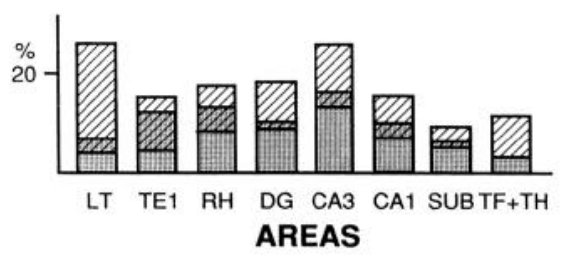

Different responses to large and small stimuli for S2 presentations

Different responses to large and small stimuli for $\mathrm{S} 1$ presentations Area of overlap:

7 different responses to large and small stimuli for S1 and S2

Figure 6. The proportions (percent of total number of recorded neurons) of differentially responsive neurons in the eight areas sampled. a, Proportions for response to S1 being significantly different from that to $\mathrm{S} 2$. $b$. Proportions for response to square stimuli being significantly different from that to nonsquare stimuli for S1 or S2 (the area of overlapped shading and hatching in the histogram represents the proportion with changes for $\mathrm{S} 1$ and $\mathrm{S} 2$; the total proportion, for $\mathrm{S} 1$ and/or S2, is given by the full height of the bars). The proportions of neurons showing such differences varied significantly between the areas for responses to S1 (BEM $\chi^{2}=57.3 ; \mathrm{df}=7 ; P<0.001$ ), to S2 (BEM $\chi^{2}=34.2 ; P<0.001$ ), and to both S1 and S2 (BEM $\left.\chi^{2}=32.2 ; P<0.001\right)$. In each case, the difference was based on a higher proportion of differentially responsive TE+RH than HF+PHG neurons (TE+RH to HF+PHG: for S1, 34.1\% to $9.6 \%$; for S2, 30.2\% to $14.2 \%$; for S1 and S2, $20.2 \%$ to $4.9 \%$ ). $c$, Proportions for response to small stimuli being significantly different from that to large stimuli for S1 or S2 (indicated as in $b$ ). These proportions did not vary significantly between the various areas. The proportion for which the difference was present for both S1 and S2 did vary significantly between the various areas $\left(\mathrm{BEM} \chi^{2}=14.58 ; \mathrm{df}=7 ; P<0.05\right)$; the proportion $(6.2 \%)$ of $\mathrm{TE}+\mathrm{RH}$ neurons was significantly higher than that $(1.8 \%)$ of $\mathrm{HF}+\mathrm{PHG}$ neurons. 


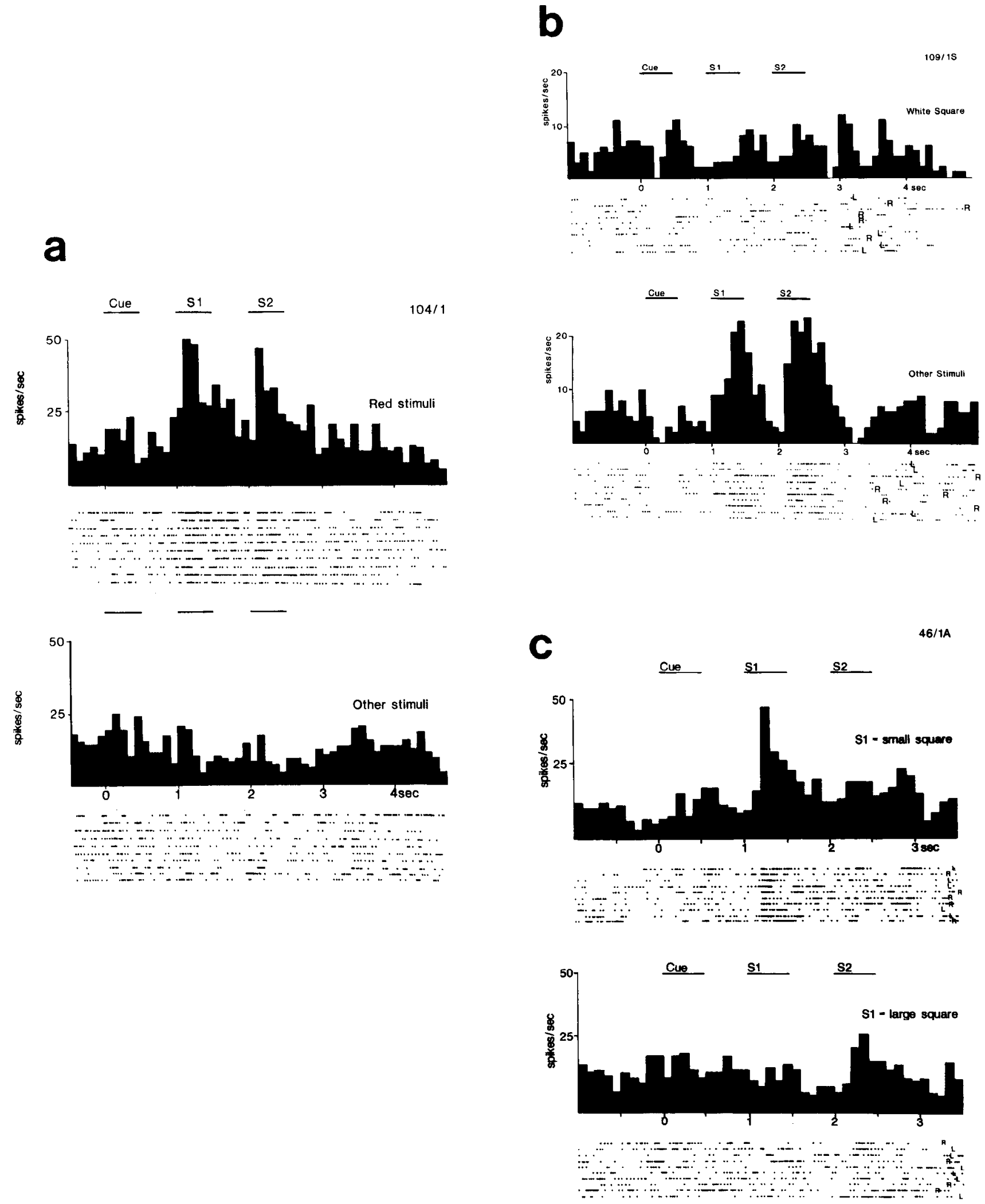

Figure 7. Examples of selectivity of neuronal response to stimulus color, shape, and/or size. $a$, Histograms and rasters of a neuron, recorded in TE1, that responded significantly more to a variety of red-colored stimuli presented as S1 and S2 (upper histogram and rasters) than to other stimuli (lower histogram and rasters); in each case, responses to 10 different pairs of stimuli are shown. There was little response to the cue, though it was also red. Thus, color alone did not fully determine the neuronal response. Conventions are as in Figure 4. $b$, Activity of a neuron, recorded in TEl, that responded significantly less to white squares (10 trials shown in upper histogram and rasters) than to stimuli of various other colors and shapes (a trial for each of 10 different pairs shown in lower histogram and rasters). $c$, Activity of a neuron, recorded in CA3, that responded significantly more to $\mathrm{S} 1$ when $\mathrm{S} 1$ was a small white square (upper histogram and rasters) than when it was a large white square (lower histogram and rasters). 
Table 2. The number of neurons with responses that declined significantly upon stimulus repetition, the total that responded to visual stimulation, and the total tested in the various regions, for neurons recorded during performance of the delayed matching task

\begin{tabular}{lccccccrc} 
& LT & TE1 & RH & DG & CA3 & CA1 & SUB & TF+TH \\
\hline Decline & 1 & 3 & 8 & 0 & 0 & 0 & 0 & 0 \\
Decline/responsive (\%) & 5.9 & 5.9 & 33.3 & 0 & 0 & 0 & 0 & 0 \\
Decline/total (\%) & 3.7 & 3.8 & 19.5 & 0 & 0 & 0 & 0 & 0 \\
Responsive & 17 & 51 & 24 & 31 & 5 & 27 & 44 & 38 \\
Total & 27 & 80 & 41 & 61 & 20 & 52 & 105 & 54
\end{tabular}

Not all recorded neurons were tested for response decrement. Nineteen neurons in TE + RH were tested only for overall responsiveness and possible response decrement; their data are not included in Table 1 or in Responses to delayed matching task in Results.

given in Table 3. The proportion of responsive neurons varied significantly between the various areas $\left(B E M \chi^{2}=41.8 ; \mathrm{df}=7\right.$; $P<0.001)$. The difference was due to the proportion of responsive HF neurons ( 31 of $85 ; 36.5 \%$ ) being significantly lower than that for the remaining areas (113 of $186 ; 60.8 \%)$. These results parallel those found for responsiveness in the delayed matching task, with the exception of the comparatively small sample of TF + TH neurons ( 15 of $24 ; 62.5 \%$ responsive). Also consistent with the findings from the delayed matching task, neurons commonly responded more to some stimuli than to others (Fig. 10); such selectivity was not systematically explored.

\section{Changes in response when unfamiliar stimuli were repeated}

For 21 neurons responses were found to be maximal to the first presentation of objects that had not been seen recently and then to decline significantly (paired $t$ tests) when the stimuli were shown subsequently. The locations of these neurons are shown in Figure 9, and an example is illustrated in Figure $8 b$. Their incidence in the individual areas is given in Table 3. The incidence of such decremental responses varied significantly(BEM $\left.\chi^{2}=37.4 ; \mathrm{df}=7 ; P<0.001\right)$ between the different areas. The proportion of neurons with such decremental responses (17 of $80 ; 21.3 \%)$ was highest in TE1, being significantly $(P<0.01)$ more than that $(4$ of $66 ; 6.1 \%$ ) in $\mathrm{RH}$, which in turn was significantly $(P<0.01)$ more than the total absence of such responses found in the other areas ( 0 of $125 ; 0 \%)$. These findings parallel those for the delayed matching task, with the exception of the comparatively small sample of LT neurons ( 0 of 16 declining responses to objects) and the reversal of the relative frequency of occurrence of declining responses in TE1 and RH. However, when expressed as a proportion of the number of responsive neurons, the incidence of neurons with declining responses does not differ significantly between TE1 and RH, though these proportions still differ significantly $(P<0.01)$ from those in $\mathrm{HF}+\mathrm{PHG}$.

Eleven of the neurons whose responses to objects declined with stimulus repetition were also recorded during the delayed matching task: the responses of five $(46 \%)$ were shown to decline to the geometrical stimuli presented during the behavioral task. It should be noted that the tested neurons often responded to only a proportion of the presented stimuli. Accordingly, some failures to establish statistically that a neuron's response declined with stimulus repetition were due to the absence of responses to the first (as well as to subsequent) presentations of some stimuli.

\section{Persistence of response decrement}

There were sufficient data for seven of the neurons with decremental responses (five in TE1, two in RH) to test the significance of any response decrement remaining after intervening presentations of other objects (see Fig. $8 b$ ). For six (86\%) of the neurons (four in TE1, two in RH), the response did not return to its initial size (paired $t$ tests). For three of four of the neurons there was evidence that the response did not recover even when there were more than two intervening items. The maximum delay tested was $>100 \mathrm{sec}$, during which period 15 intervening items were presented and the neuronal response did not recover.

Evidence for longer-term storage of information was found by comparing the responses to unfamiliar stimuli with those to highly familiar but not recently seen stimuli for the 21 neurons whose responses declined significantly to repeated presentations of objects. The highly familiar stimuli had not been seen in the

Table 3. The number of neurons with responses that declined significantly upon stimulus repetition, the total that responded, and the total tested in the various regions, for neurons tested for responsiveness to the sight of objects

\begin{tabular}{lclcccccc} 
& LT & TE1 & RH & DG & CA3 & CA1 & SUB & TF+TH \\
\hline Decline & 0 & 17 & 4 & 0 & 0 & 0 & 0 & 0 \\
Decline/responsive (\%) & 0 & 26.6 & 16.7 & 0 & 0 & 0 & 0 & 0 \\
Decline/total (\%) & 0 & 21.3 & 6.1 & 0 & 0 & 0 & 0 & 0 \\
Responsive & 10 & 64 & 24 & 3 & 2 & 9 & 17 & 15 \\
Responsive/total (\%) & 62.5 & 80.0 & 36.4 & 15.8 & 40.0 & 36.0 & 47.2 & 62.5 \\
Total & 16 & 80 & 66 & 19 & 5 & 25 & 36 & 24
\end{tabular}

Of these neurons, 71 in TE+RH and 73 in HF+PHG were also recorded during the delayed matching task; five had responses that declined in both situations (see Results). Thus, in total, 28 (12\%) of 239 TE + RH neurons had decremental responses, whereas none (0\%) of $328 \mathrm{HF}+$ PHG neurons did. 


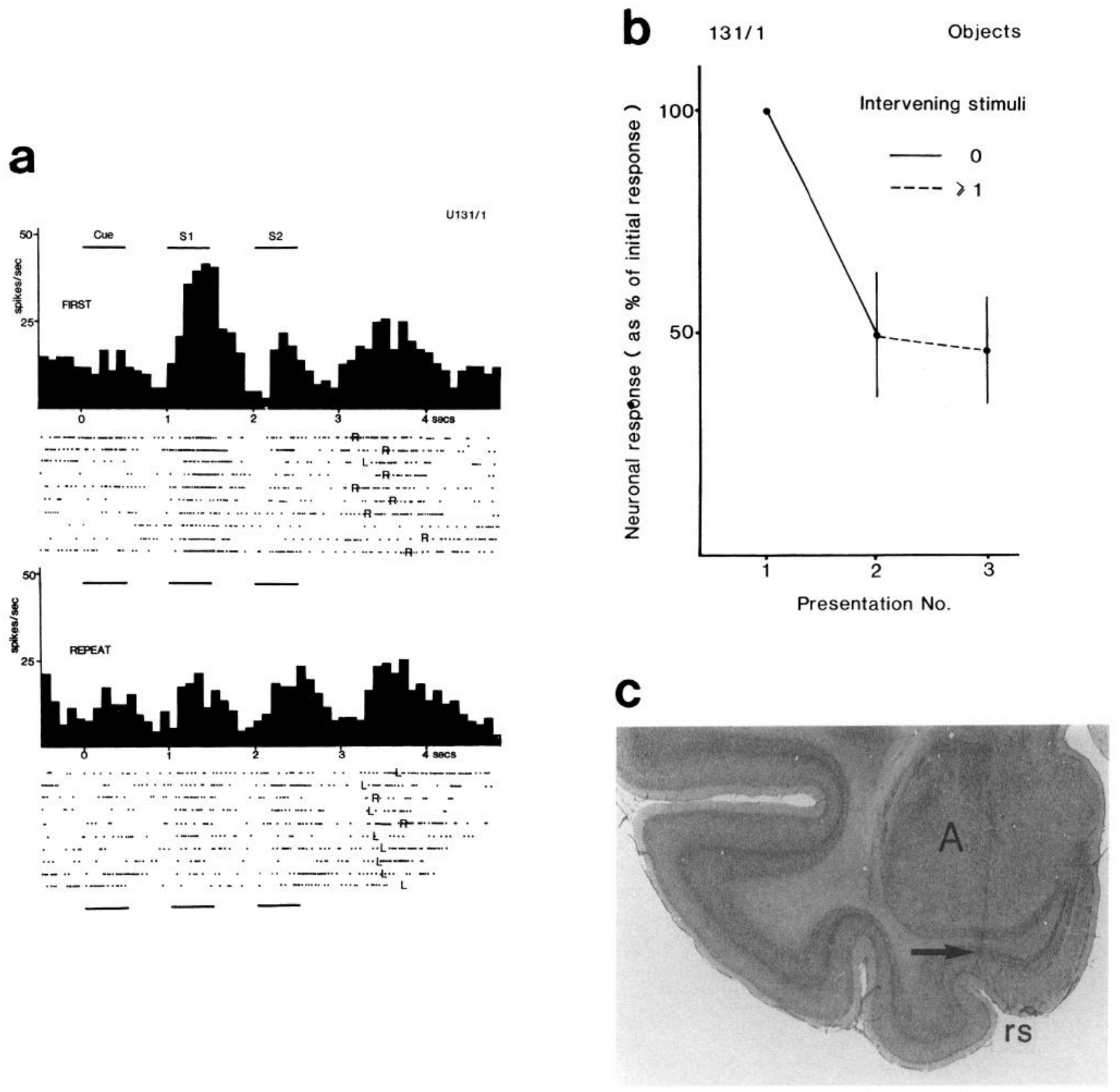

Figure 8. Example of response decrement with stimulus repetition. a, Responses of a neuron (U131/1) to the first (upper histogram and rasters) and second presentations (lower histogram and rasters) of 10 different stimuli, presented as S1, that had not been seen recently. Note the much stronger response to first than to repeat presentations of the stimuli. $b$, The decline in response of the same neuron $(U 131 / 1)$ to repeated presentations of objects (mean \pm SEM for second and third presentations; response to first presentation $=100 \%$ ). Note that the response does not recover even when there are intervening presentations of other objects. $c$, Electrode track with reconstructed position (indicated by an arrow) in prorhinal cortex at which neuron $\mathrm{U} 131 / 1$ was recorded. $A$, amygdala; $r$, rhinal sulcus.

great majority of cases for the previous $15 \mathrm{~min}$ or more. For seven (33\%) neurons, responses to the first presentations of unfamiliar stimuli were significantly (two-sample $t$ tests) greater than to the first presentations of highly familiar stimuli (e.g., Fig. 11): for no neurons were the responses to familiar stimuli significantly greater than those to unfamiliar stimuli. Indeed, the mean response to the first presentations of unfamiliar objects was greater than that to the first presentations of highly familiar objects for $17(81 \%)$ of the 21 neurons; this proportion is significantly higher than the $50 \%$ expected by chance (binomial test, $P<0.01$ ). Similarly, the mean response to first presenta- tions of unfamiliar objects was greater than the mean response to sight of the highly familiar fluid-filled syringe for 18 of 21 $(86 \%)$ of the neurons and greater than the mean response to pieces of food for 20 of 21 (95\%) of the neurons (both proportions greater than that expected by chance; $P<0.001$ ).

\section{Other responses}

Although responses to stimuli of other modalities were not systematically sought in these experiments, seven neurons (five in $\mathrm{HF}+\mathrm{PHG}$, two in $\mathrm{RH}$ ) were clearly responsive to auditory stimulation. Five $(71 \%)$ of these seven neurons (three in HF+PHG, 


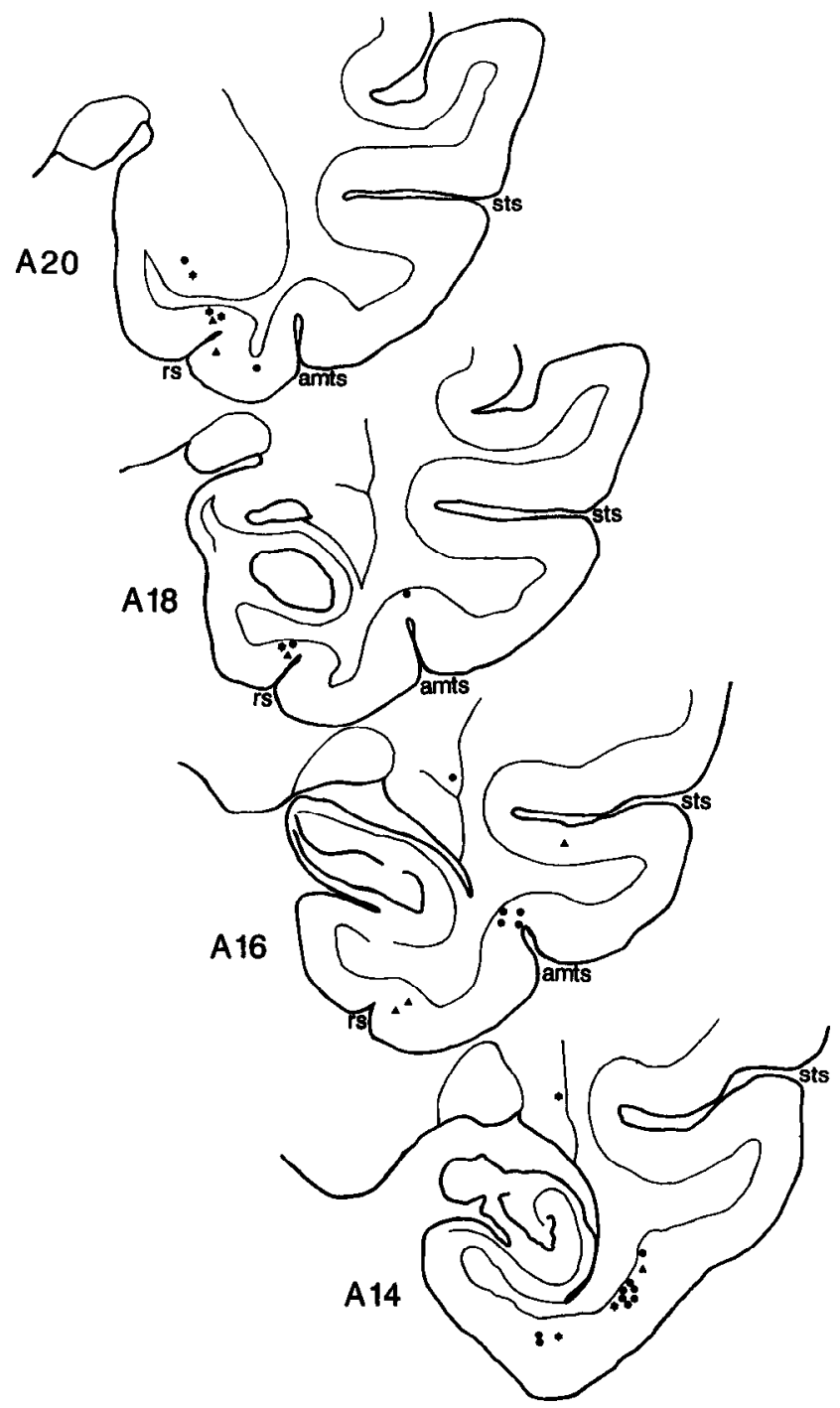

Figure 9. The locations of neurons with decremental responses in the delayed matching task (triangles), to the sight of objects (circles), or in both situations (asterisks). For details, see Figure 5.

two in $\mathrm{RH}$ ) were also responsive to visual stimuli. Certain neurons responded to specific categories of stimuli: one neuron in TE1 responded selectively to the sight of foods; another TEl neuron, to human faces; and a neuron in TG, only to the sight of the juice-filled syringe.

\section{Other areas}

During the course of these experiments, small populations of neurons were recorded in neighboring structures. Of 28 neurons recorded in the basolateral amygdala, nine (32\%) were visually responsive; two $(7 \%)$ neurons had responses that declined upon stimulus repetition. Of 20 neurons recorded in the ventral putamen, $11(55 \%)$ were visually responsive; two (10\%) neurons had responses that declined when stimuli were repeated.

\section{Discussion}

The results indicate that there are both similarities and differences in neuronal responsiveness between the HF and the neighboring cortical regions with which it is connected: cortex adjacent to the rhinal sulcus, inferior temporal cortex (recordings

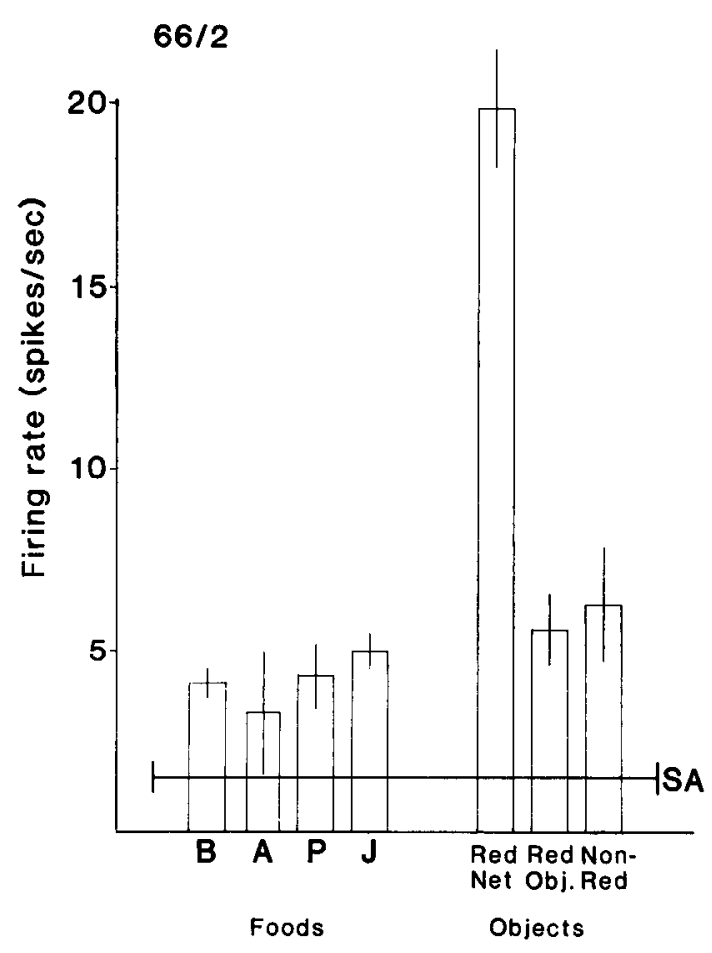

Figure 10. Example of selectivity in neuronal response to sight of objects. The neuron, recorded in area TF, responded selectively to the sight of a small red net. Neurons that responded to only a very restricted subset of stimuli were also encountered using the delayed matching task. Data are mean ( \pm SEM) response to the sight of the small red net, other red and nonred objects, and foods ( $B$, banana; $A$, apple; $P$, peanut; $J$, syringe filled with blackcurrant juice). $S A$, mean \pm SEM spontaneous activity.

were made mainly from its medial and anterior part), and areas TF and TH. The similarities include the findings that (1) neurons in all areas were responsive to the visual stimuli presented in the delayed matching task (cue, S1 and S2); (2) neurons in all areas were responsive to the sight of objects; (3) a subpopulation of the responsive neurons were selectively responsive to particular stimuli; (4) the magnitudes of the mean responses to the visual stimuli were similar in the different areas; and (5) similar proportions (16\%) of neurons were sensitive to differences in the size of the stimuli used as S1 and S2. In addition, Wilson et al. (1990) have shown that the presence of activity related to the behavioral response of the monkey is a common feature of neurons in all these areas.

Both qualitative and quantitative differences between the regions were also found. These differences were chiefly between the inferior temporal and rhinal cortex on the one hand and areas $\mathrm{TF}$ and $\mathrm{TH}$ and the $\mathrm{HF}$ on the other. No major difference between the HF and areas TF and TH was evident in these experiments. The most notable, qualitative difference between the areas was the presence in the inferior temporal and rhinal cortical regions of a population of neurons whose responses showed a large decrement from the first to subsequent presentations of stimuli that had not been seen recently; such neurons were not found in areas TF or TH or in the HF. The statistically significant quantitative differences included (1) the proportion $(30 \%)$ of neurons responding with an increase in activity to the cue in area TE1 and rhinal cortex was three times that (10\%) for the remaining areas; (2) the proportion of neurons respond- 


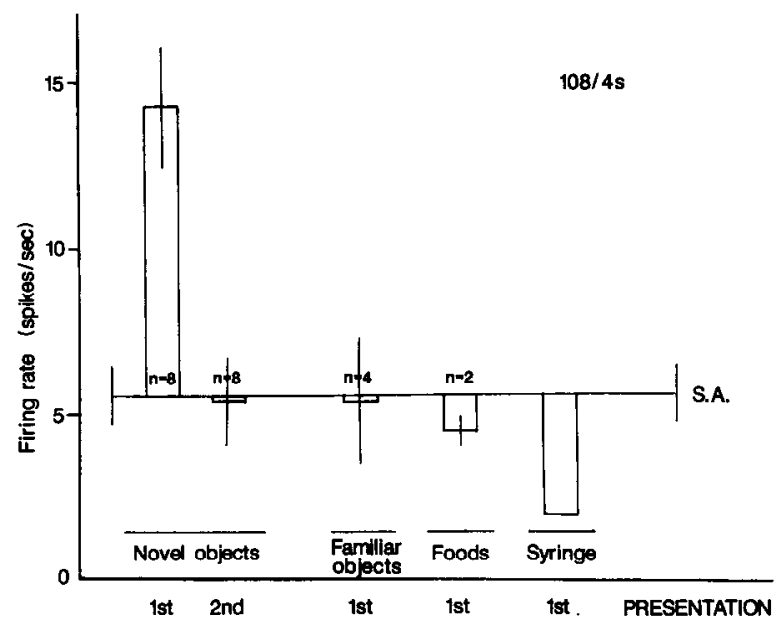

Figure 11. Example of a neuron, recorded in TE1, responding more strongly to novel than to familiar objects. Mean \pm SEM responses are shown. The mean response to the first presentations of novel objects was significantly greater than that to the second presentation of these objects, but also significantly greater than that to first presentations of highly familiar objects, familiar foods, or the familiar syringe containing fruit juice. The familiar objects had not been seen in the previous $>15$ min. $S . A$., mean \pm SEM spontaneous activity.

ing to $\mathrm{S} 1$ and/or $\mathrm{S} 2$ in the inferior temporal and rhinal cortical regions (TE+RH neurons) was higher (65\%:53\%) than in either the HF or areas TF and TH of the PHG (HF+PHG neurons); (3) the proportion of $\mathrm{TE}+\mathrm{RH}$ neurons responding to the sight of objects $(61 \%)$ was similar to that found for S1 and S2, but the proportion of hippocampal neurons responding to the sight of objects was much lower (37\%); (4) the proportion $(44 \%)$ of $\mathrm{TE}+\mathrm{RH}$ neurons whose response changed when the shape of the stimulus used as $S 1$ and/or $S 2$ changed was more than twice as high as that (19\%) of HF+PHG neurons; and (5) of the neurons that responded differently depending on whether stimuli were presented as $\mathrm{S} 1$ or $\mathrm{S} 2,66 \%$ of such $\mathrm{TE}+\mathrm{RH}$ neurons responded more strongly to $\mathrm{S} 1$, while $71 \%$ of such $\mathrm{HF}+\mathrm{PHG}$ neurons responded more strongly to $S 2$.

The effects of visual stimulation and memory upon the responses of medial temporal neurons will be further considered before implications of the findings for understanding the functioning of the region are addressed.

\section{Visual stimulation}

Responses to the cue

In all regions (though most notably in the HF), there was a paucity of neurons responsive to the visual cue that signaled the start of a trial in the delayed matching task, relative to the incidence of neurons responsive to the two visual stimuli (S1 and S2) that were to be compared. Further, the magnitude of the response to the cue was typically smaller than that to $S 1$ and $\mathrm{S} 2$. These differences may have arisen because in two regards the information carried by the cue was less than that for $\mathrm{S} 1$ and S2. First, the cue consisted of diffuse illumination of the screen for $0.5 \mathrm{sec}$ and was therefore a relatively unstructured visual stimulus compared to the geometrical patterns used as S1 and S2. Second, the cue did not convey information other than to signal the beginning of a trial and hence did not require extensive analysis, unlike $\mathrm{S} 1$ and $\mathrm{S} 2$, whose details had to be remembered. The present data are consistent with findings in lateral and more posterior parts of inferior temporal cortex (which provide inputs to the medial temporal region), where neurons do not respond as well to flashes of light as to highly structured stimuli (Desimone et al., 1984). However, they contrast with findings in the basal forebrain, including the vertical limb of the diagonal band of Broca (which also projects to the medial temporal region), where many neurons are as responsive to an auditory cue signaling the start of a trial as to the visual stimuli signaling which behavioral response to make (Wilson and Rolls, 1990b). The difference in incidence of responses to the cue between the various areas will be commented on below.

It is possible that at least some responses to the cue simply reflected an increase in the level of alertness of the animal with the commencement of a trial: in subprimate species, alterations in arousal have been shown to produce changes in the activity of hippocampal neurons (Mays and Best, 1975; Brown and Horn, 1977). Changes in arousal or the anticipation of reward might contribute to the neuronal responses to not only the cue but also to the stimuli presented as S1 and S2. However, there was no evidence for gross changes in the level of arousal of the animals during performance of the task. Furthermore, for example, responses to $S 1$ and $S 2$ that are dependent on the particular visual stimuli presented cannot simply be explained by arousal changes. It should also be noted here that videomonitoring of eyc movements did not reveal any close relationship between eye movements and neuronal activity.

\section{Overall responses to $S 1$ and $S 2$}

More than half of the neurons in both the HF and its neighboring cortex responded to the visual stimuli ( $\mathrm{S} 1$ and $\mathrm{S} 2$ ) used in the delayed matching task. The incidence may have been influenced by the animals' training, especially for HF neurons: in the HF the incidence of responses to the stimuli used as $\mathrm{S} 1$ and $\mathrm{S} 2$ was considerably higher than that to the sight of objects that had not been used in the training of the animals, whereas the proportions for $\mathrm{TE}+\mathrm{RH}$ were approximately equal. The effects of training upon neuronal responsiveness in the medial temporal lobes has been noted in other studies (Fuster and Uyeda, 1971; Miyashita, 1988). The incidence of responsive neurons in TE + RH may have been slightly underestimated because of the tendency of the responses of certain neurons to decline upon stimulus repetition. However, the magnitude of the underestimate seems likely to be only about $3 \%$ because $12 \%$ of all TE $+\mathrm{RH}$ neurons had responses that declined significantly and the overall mean response in the delayed matching task was found to be nonsignificant for only one-quarter of such neurons.

The high incidence of responsive neurons presents a problem for the theoretical modeling of memory: typically, a very low proportion $(<1 \%)$ of responsive cells is required if rapid saturation of modifiable synapses is to be avoided (e.g., Marr, 1971; Gardner-Medwin, 1976). However, the findings of the present study are consistent with previous reports of high proportions of neurons responding during the performance of various behavioral tasks employing but a limited range of sensory stimuli, both in the HF and in lateral parts of the inferior temporal cortex (Fuster and Uyeda, 1971; Vinogradova, 1975; Berger and Thompson, 1978; Gross et al., 1979; Mikami and Kubota, 1980; Watanabe and Niki, 1985; Heit et al., 1988; Miyashita et al., 1989; Rolls et al., 1989). The present study extends these results to the more medial inferior temporal cortex and areas TF and TH. High incidences of responsive medial temporal neurons to neutral (i.e., nonconditioned) sensory stimuli have been re- 
ported in other studies, as well (e.g., Fuster and Uyeda,1971; Vinogradova, 1975; Brown and Horn, 1977).

\section{Selective responses to visual stimuli}

Evidence for the differential encoding of physical attributes of the stimuli (color, shape, and/or size) was found for $29 \%$ of the neurons: the activity of these neurons changed significantly when the stimulus was changed in the delaycd matching task. The most common discrimination required for performance of the delayed matching task was of the relative size of the stimuli shown as $\mathrm{S} 1$ and $\mathbf{S 2}$. Thus, if training were the major determinant of neuronal responsiveness, it might be expected that the great majority of these differentially responsive neurons would have been sensitive to changes in the size of the stimuli. In fact, the activity of $16 \%$ of the neurons (the proportion did not vary significantly between areas) varied with the size of stimuli. However, size discrimination is little affected by inferior temporal lesions (Humphrey and Weiskrantz, 1969; Ungerleider et al., 1977). Morever, in LT the majority of neurons have been found to be insensitive to changes in size of the stimuli (Desimone et al., 1984). The shape and/or color of the visual stimuli used as $\mathrm{S} 1$ and $\mathrm{S} 2$ influenced the responses of $19 \%$ of the $\mathrm{HF}+\mathrm{PHG}$ neurons. In contrast, the response of more than twice (44\%) the proportion of TE I RH neurons changed when the stimulus changed from being a white square. This change in mean response to stimuli is likely to be due to a sensitivity to stimulus shape and/or color rather than to the relative novelty of the stimuli because the proportion of differentially responsive neurons that responded preferentially to the white square was approximately the same $(\approx 39 \%)$ for $\mathrm{HF}+\mathrm{PHG}$ neurons, for TE+RH neurons, and furthermore, even for those TE + RH neurons that had decremental responses. The sensitivity to stimulus change ranged from graded alterations in the magnitude of the responses to different stimuli (e.g., responses in Fig. 8a), to responses to only one of several stimuli used in testing (Fig. 10). Certain TE $+\mathrm{RH}$ neurons were selectively responsive to stimuli of a particular color (Fig. 7a). LT neurons have been shown previously to encode information about the color and shape of stimuli (Gross et al., 1979; Desimone et al., 1984; Baylis et al., 1987). Thus, consistent with findings in LT, neurons of more anterior and medial temporal cortex also signal information about the identity of visual stimuli. However, influences other than the color and shape of stimuli were also important determinants of neuronal responses in these areas (see below).

\section{Differences in response to $S 1$ and $S 2$}

Certain neurons ( $18 \%$ of the total) differed in their responses to S1 (sample presentation) and S2 (match/nonmatch comparisons). Of such differentially responsive $\mathrm{HF}+\mathrm{PHG}$ neurons, $71 \%$ responded more strongly to $S 2$, that is, at the time the behavioral decision to go left or right could be made. The importance of the medial temporal region for the selection of spatially directed behavioral responses has been discussed by Wilson et al. (1990). In contrast, $66 \%$ of such differentially responsive TE $+\mathrm{RH}$ neurons responded more strongly to the sample presentation (S1), that is, at acquisition. Inspection of Figure $4, a$ and $c$, will show that changes in eye movements do not provide a general explanation for differences in response to $S 1$ and $S 2$, because there were no differences in eye movements between $S 1$ and $S 2$ during the first $250 \mathrm{msec}$ after stimulus onset. Hence, the results may be taken as indicating a role in mnemonic processes for such differentially responsive neurons. However, this role should be distinguished from that of the TE $+\mathrm{RH}$ neurons whose responses decline when a stimulus that has not been seen recently is repeated. The difference in mean responses between TE $+\mathrm{RH}$ and $\mathrm{HF}+\mathrm{PHG}$ neurons is not due primarily to the decline in response of such TE + RH neurons: of the TE + RH neurons that responded more strongly to $\mathrm{S} 1$ than $\mathrm{S} 2$, only $26 \%$ were neurons with responses that declined significantly upon stimulus repetition; for the remainder, the order of the trials in a block had no consistent effect on responses to $\mathrm{S} 1$ and $\mathrm{S} 2$ (cf. Figs. $4 a, 8 a$ ). Accordingly, there appears to be more than one type of neuronal activity within TE $+\mathrm{RH}$ that may be linked to memory processes.

\section{Hippocampal regional differences}

The proportion of neurons responsive to $\mathbf{S} 1$ and/or $\mathbf{S} 2$ was higher in the posterior than in the anterior HF (60\%:44\%). The proportion of neurons responding differently to $\mathrm{S} 1$ than to $\mathrm{S} 2$ was over twice as high in the posterior as in the anterior $\mathrm{HF}$ (22\%:8\%). Similarly, a higher incidence of neurons responding differently on go-left than on go-right trials (Wilson et al., 1990) and of "delay" neurons (Watanabe and Niki, 1985) has been found in the posterior than in the anterior HF. These findings may reflect the topographical organization of inputs to the HF (Witter et al., 1989).

\section{Memory \\ Decremental responses}

A statistically significant decrement in neuronal response with stimulus repetition either in the delayed matching task or to the sight of objects was found for $12 \%$ of the neurons (18\% of the visually responsive neurons) recorded in $\mathrm{TE}+\mathrm{RH}$, but for no neurons in $\mathrm{HF}+\mathrm{PHG}$. Furthermore, a population measure (comparison of first responses to mean responses to stimuli in the delayed matching task), based upon all the TE+RH neurons responding with an increase in activity, also evidenced a decline in response upon the repeated presentation of stimuli, but this was not so in $\mathrm{HF}+\mathrm{PHG}$. The influence upon a population measure emphasizes the magnitude/prevalence of such response decrement in $\mathrm{TE}+\mathrm{RH}$. The largest decrements in response were invariably between the first and second presentations of stimuli. With further repetitions, the response did not necessarily decline to 0 , so that the response averaged across all presentations could still be significant, though submaximal. The response decrements were found for stimuli that were being used to obtain reward and persisted after presentations of other distracting or alerting stimuli. They may therefore be distinguished from those of classical short-term habituation (Thompson and Spencer, 1966). Whether the synaptic mechanism underlying such response decrements is similar to either that of habituation or that of long-term depression remains to be determined (Kandel, 1981; Ito, 1989).

Such response decrements must be an endogenous property of the region because they occur to stimuli (the sight of objects) that had not been used in the training of the animals. Such decrements were also found when stimuli that had not been seen recently were introduced during performance of the delayed matching task. Because the monkeys continued to perform the task and obtain reward, gross changes in arousal and attention may be excluded as an explanation of the decrements in neuronal response. Videorecordings of eye movements and behavior revealed no changes paralleling the neuronal response decrements and indicated that examination of the visual stimuli 
was maintained during the periods for which the data were analyzed. We have subsequently verified, using the subscleral search-coil technique (F. A. W. Wilson and P. S. GoldmanRakic, unpublished observations), that the latencies, durations, and frequency of saccadic eye movements during the period of data collection are not altered by changes in stimulus familiarity and so cannot account for the differential neuronal responses described here. An additional argument against the decrements in neuronal responses being due to peripheral, ocular changes is that such decrements were not found for visually responsive neurons recorded in the HF or areas TF and TH.

No similar response decrement with stimulus repetition was found for neurons in areas TF and TH and the HF either using the delayed matching task or when objects were shown to the monkey. Moreover, therc was no significant difference between mean responses and responses to the first presentations of visual stimuli in the delayed matching task in these hippocampal areas. A corresponding absence of responses varying with the novelty or familiarity of stimuli was found for the HF during performance of a go/no-go serial recognition task (Wilson et al., 1988, 1990): 295 neurons were recorded from three monkeys during the recognition task. Rolls et al. (1989) found that $0.4 \%$ (4 of 994) of hippocampal neurons responded more to novel than to familiar stimuli in a similar recognition task, and in a task requiring memory for where a stimulus appeared, $2.4 \%$ (24 of 994) of hippocampal neurons responded more to novel stimuli, but only if they were presented in a particular place. Thus, hippocampal neurons are rarely responsive to the relative novelty or recency of presentation of stimuli per se.

\section{Persistence of decrement and familiarity discrimination}

For certain neurons it was possible to establish that the response decrement persisted across an interval filled by presentations of other stimuli that would have distracted the animal's attention. The maximum interval between two presentations of a object for which a neuronal response decrement was found was 15 intervening presentations of other stimuli, representing a delay of over $100 \mathrm{sec}$. For some neurons there was evidence suggesting that the interval over which information concerning the previous presentation of stimuli could be maintained was of at least some minutes duration. For $81 \%$ of the neurons with responses that declined, the response to first presentations of unfamiliar objects was stronger than that to first presentations of very familiar objects, and for $33 \%$ of the neurons there were sufficient data to show that this difference was significant. The familiar objects had typically not been seen during the previous $15 \mathrm{~min}$ or more, suggcsting that the fact of their previous occurrence could be signaled by neuronal activity after storage for at least that length of time. These findings also suggest that at least some neurons in this region may signal information concerning the relative novelty or familiarity of the stimuli. However, the present data do not allow clear separation of the possible effects of memory persistence over long periods from those of the relative familiarity of the stimuli. Moreover, the responses of the neurons were also influenced by the physical features of the stimuli. Because it is not possible to know that the physical attributes of the familiar and unfamiliar stimuli were equivalent in their potential to excite the neurons, any conclusions must be tentative at present. However, the fact that for none of the neurons were the responses to familiar objects significantly greater than to unfamiliar objects is supportive of the suggestion. For other neurons with decremental responses, the data indicated that response decrements were not maintained over long intervals, so that the neurons of this region may display a range of different mnemonic capacities. It remains to be established that such neurons can signal information concerning the previous occurrence of stimuli over periods that are sufficiently long to explain the full abilities of monkeys in recognition memory tasks (Ringo and Doty, 1985).

The decremental responses should be contrasted with the sustained activity displayed by some inferior temporal neurons in tasks that require the occurrence of a particular, highly familiar stimulus be maintained in memory across an unfilled (undistracted) interval of some seconds (Fuster and Jervey, 1982; Miyashita, 1988). Such sustained activity could reflect an attentive or short-term memory mechanism: however, the response decrements of the present study were maintained even after attention had been distracted. The sustained activity provided an appropriate mechanism for solving the specific tasks to which the animals were trained: the decremental responses were independent of the animals' training or task performance and may thus play a more general mnemonic role in signaling the familiarity or recency of presentation of stimuli. These results provide further evidence that the inferior temporal cortex may be involved in more than one type of memory process.

\section{Relation to lesion studies}

Evidence from neuropathological studies of humans suggests that the medial temporal lobes contribute to recognition memory (Parkin, 1987; Squire, 1987; Mayes, 1988). However, patients with medial temporal lobe lesions that include at least parts of the cortex near the rhinal sulcus, in spite of having poor recognition memory, show essentially normal enhancement of performance by prior experience, that is, intact priming (Milner, 1970; Shimamura, 1986; Schacter, 1987). This preserved priming in the human reduces the likelihood that the neurons with decremental responses in this cortex in the monkey are necessary for priming memory. Cooling of the anterior inferior temporal gyrus disrupts monkeys' performance of a task requiring memory for the previous occurrence of stimuli (Horel et al., 1987). Moreover, two recent studies have indicated that monkeys with lesions including cortex near to the rhinal sulcus, but excluding the hippocampus and amygdala, are severely impaired in recognition memory tasks (Murray et al., 1989; Zola-Morgan et al., 1989). The data from the lesion and cooling studies taken in conjunction with the data about neuronal responses from the present experiments make a strong case for the importance of the cortex close to the rhinal sulcus to recognition memory.

\section{Associated areas}

TE1 and rhinal cortex receive major inputs from the more laterally placed inferior temporal cortex (Van Hoesen and Pandya, 1975). In previous studies of lateral and posterior inferior temporal cortex, there has been mention of neurons whose responses "habituated" with stimulus repetition (Gross et al., 1972; Richmond and Sato, 1987). Baylis and Rolls (1987) have shown that the great majority of neurons whose responses declined to repeated presentations of visual stimuli were unable to maintain their response decrement across even one intervening presentation of another stimulus and never across more than two intervening presentations. There was no evidence for the one neuron with a declining response recorded in LT in the present experiments that the decrement persisted if there were intervening trials of other stimuli. Hence, the persistence of the re- 
sponse decrements of at least some medial inferior temporal and rhinal cortical neurons is not a simple reflection of the region's inputs from $\mathrm{LT}$. This finding is again consistent with the suggested importance of the more anterior and medial temporal cortex for memory.

In addition to its connections with the $\mathrm{HF}$, the anterior and medial inferior temporal, and rhinal cortex sends projections to the amygdala, the ventral putamen, the basal forebrain, and the periventricular region of the diencephalon (Aggleton et al., 1980; Van Hoesen et al., 1981; Russchen et al., 1985; Wilson and Rolls, 1990a). Neurons that respond on the basis of the novelty or familiarity of visual stimuli have been found in each of these regions (Rolls et al., 1982; Wilson and Rolls, 1987, 1990a; present results). The medial inferior temporal and rhinal cortex also sends fibers to the medial thalamus and to the inferior and medial prefrontal cortex (Aggleton et al., 1986; Yeterian and Pandya, 1988; Ungerleider et al., 1989); there are no reports indicating whether neurons in these regions signal information concerning the prior occurrence of stimuli. However, lesion studies have implicated the medial thalamus and the inferior and medial prefrontal cortex as well as the medial temporal lobes and basal forebrain in the processes necessary for the performance of recognition memory tasks (Aggleton and Mishkin, 1983; Bachevalier and Mishkin, 1986; Aigner et al., 1987). Thus, there is anatomical, neuropsychological, and electrophysiological evidence linking structures involved in recognition memory with the medial inferior temporal and rhinal cortex.

\section{What do medial temporal neuronal responses represent?}

The data indicate that the responses of a number of distinct categories of medial temporal neurons do not reflect solely the physical properties of the stimuli. The clearest example is that provided by the neurons whose responses decremented upon stimulus repetition. A second example is given by those neurons for which the response was dependent upon whether a stimulus was presented as $\mathrm{S} 1$ or S2. A third indication is that for $\mathrm{HF}$ neurons there was a marked difference in the proportion responsive to the visual stimuli presented in the delayed matching task (which wcrc cach of a uniform color, texture, and brightness) and the lower proportion responsive to the much more varied stimuli, but with no reinforcing associations, presented outside the task. Thus, neuronal responses in these regions may reflect abstract features of the task such as the difference in behavioral meaning of stimuli presented as S1 or S2. Such findings are consistent with the deficits in performance of delayed matching tasks found in lesion or cooling studies (Stepien et al., 1960; Correll and Scoville, 1965; Mishkin, 1978; Mahut et al., 1982; Malamut et al., 1984; Zola-Morgan and Squire, 1985; Horel et al., 1987; Zola-Morgan et al., 1989). Furthermore, for many medial temporal neurons, changes in activity occur in relation to the behavioral responses in the delayed matching task and may be involved in the selection of these behavioral responses (Wilson et al., 1990).

The present results reveal a tendency for hippocampal neurons to be less visually responsive than those of the inferior temporal and rhinal cortex, except in certain task-related aspects. Thus, the hippocampal neurons were relatively less responsive to the cue and in overall response to $S 1$ and $S 2$, including the encoding of particular shapes and colors, features that were relatively unimportant for performance of the delayed matching task. They were unresponsive to the familiarity and/ or recency of the stimuli, a dimension that was irrelevant for task performance. Moreover, they were less often responsive to the sight of objects (though compared to the geometrical stimuli used in the delayed matching task, the objects were more varied in physical characteristics such as shape, color, and size) where there was no necessary behavioral contingency. However, for certain features more closely related to performance of the delayed matching task, the relative responsiveness of hippocampal neurons was increased. Thus, the incidence of neurons that responded differently to a change in size of the stimuli was as high as in other areas. Also, the incidence of neurons more responsive to $\mathbf{S} 2$ than $\mathrm{S} 1$ was higher than in other areas (the occurrence of $\mathrm{S} 2$ allowed the decision about the behavioral response to be made). However, it should not be concluded that training on any task will result in hippocampal neuronal responsiveness that reflects all features of the task; the absence of hippocampal neurons sensitive to the relative novelty of stimuli in a go/nogo recognition task (Wilson et al., 1988, 1990) provides such a counterexample.

The counterpart of the above pattern of hippocampal responsiveness was that neuronal responsiveness in rhinal and inferior temporal cortex was relatively more often closely linked to the physical features of the visual stimuli. However, the activity of some neurons was dominated by the prior history of presentation of the stimuli. Because neurons with decremental responses were typically also sensitive to physical characteristics of the visual stimuli, they could form part of a system signaling the familiarity and/or recency of presentation of individual stimulus items. The HF might therefore not be needed for such a role in recognition memory. One roie of the HF might then be to make associations between the many simultaneously experienced items that constitute a particular event, so that subsequently the event or scene characterized by the contemporaneous occurrence of these items could be remembered (Brown, 1982, 1990). One aspect of this could be the encoding of the spatial arrangement of the items: there is much evidence for the importance of memory for the spatial configuration of items to hippocampal function (O'Keefe and Nadel, 1978; Gaffan and Harrison, 1989).

In conclusion, these experiments have demonstrated that there are quantitative and qualitative differences between the responsiveness of neurons in the studied areas of the medial temporal lobe, even though these areas are closely anatomically interconnected and might be expected to be functionally integrated. All areas have been found to participate in the processing of visual information, though information concerning the physical features and hence the identity of the stimuli is more strongly represented in the anterior and medial inferior temporal and rhinal cortex than in the HF and PHG. However, the results also revealed that responses of certain neurons are not solely dependent on the physical attributes of visual stimuli. Thus, the responsiveness of hippocampal neurons displayed the influence of training and reflected particular features of relevance to the performance of the delayed matching task. Additionally, neuronal activity signaling information of importance for recognition memory was discovered: certain neurons in the anterior and medial inferior temporal and rhinal cortex signaled the familiarity and/or time of previous occurrence of visual stimuli.

Note added in proof: Heit et al. (1990) recorded neuronal activity primarily in the hippocampal formation during the performance of a recognition memory task. They found that "responses specifically to familiar (as opposed to unfamiliar) words 
or faces, or to tasks requiring recent memory per se, were never seen. Key press excitation was relatively common (32/76 units) ...."These results correspond closely to our data obtained in the monkey.

\section{References}

Aggleton JP, Mishkin M (1983) Memory impairments following restricted medial thalamic lesions in monkeys. Exp Brain Res 52:199209.

Aggleton JP, Burton MJ, Passingham RE (1980) Cortical and subcortical afferents to the amygdala of the rhesus monkey (Macaca mulatta). Brain Res 190:347-368.

Aggleton JP, Desimone R, Mishkin M (1986) The origin, course and termination of the hippocampothalamic projections in the macaque. J Comp Neurol 243:409-421.

Aigner TG, Mitchell SI, Aggleton JP, DeLong MR, Struble RG, Price DL, Wenk GL, Mishkin M (1987) Effects of scopolamine and physostigmine on recognition memory in monkeys with ibotenic-acid lesions of the nucleus basalis of Meynert. Psychopharmacology 92: 292-300.

Bachevalier J, Mishkin M (1986) Visual recognition impairment follows ventromedial but not dorsolateral prefrontal lesions in monkeys. Behav Brain Res 20:249-261.

Baker RJ, Nelder JA (1978). The GLIM (generalised linear interactive modelling) system. Oxford: Royal Statistical Society.

Baylis GC, Rolls ET (1987) Responses of neurons in the inferior temporal cortex in short term and serial recognition memory tasks. Exp Brain Res 65:614-622.

Baylis GC, Rolls ET, Leonard CM (1987) Functional subdivisions of the temporal lobe neocortex. J Neurosci 7:330-342.

Berger TW, Thompson RF (1978) Neuronal plasticity in the limbic system during classical conditioning of the rabbit nictitating membrane response. I. The hippocampus. Brain Res 145:323-346.

Brown MW (1982) Effect of context on the responses of single units recorded from the hippocampal region of behaviourally trained monkeys. In: IBRO monograph series, Vol 9, Neuronal plasticity and memory formation (Ajmone-Marsan C, Matthies H, eds), pp 557573. New York: Raven.

Brown MW (1990) Why does the cortex have a hippocampus? In: Learning and computational neuroscience: foundations of adaptive networks (Gabriel M, Moore J, eds), pp. 233-282. New York: MIT Press.

Brown MW, Horn G (1977) Responsiveness of neurones in the hippocampal region of anaesthetised and unanaesthetised cats to stimulation of sensory pathways. Brain Res 123:241-259.

Brown MW, Leendertz JA (1979) A pulse-shape discriminator for action potentials. J Physiol (Lond) 298:17P-18P.

Brown MW, Wilson FAW, Riches IP (1987) Neuronal evidence that inferomedial temporal cortex is more important than hippocampus in certain processes underlying recognition memory. Brain Res 409: 158-162.

Cassell MD, Brown MW (1984) The distribution of Timm's stain in the nonsulphide-perfused human hippocampal formation. J Comp Neurol 222:461-471.

Correll RE, Scoville WB (1965) Performance on delayed match following lesions of medial temporal lobe structures. J Comp Physiol Psychol 60:360-367.

Desimone R, Gross CG (1979) Visual areas in the temporal cortex of the macaque. Brain Res 178:363-380.

Desimone R, Albright TD, Gross CG, Bruce C (1984) Stimulus-selective responses of inferior temporal neurons in the macaque. J Neurosci 4:2051-2062.

Fox SE, Ranck JB Jr (1975) Localization and anatomical identification of theta and complex spike cells in dorsal hippocampus of rats. Exp Neurol 49:299-313.

Fuster JM, Jervey JP (1982) Neuronal firing in the inferotemporal cortex of the monkey in a visual memory task. J Neurosci $2: 361-$ 375.

Fuster JM, Uyeda AA (1971) Reactivity of limbic neurons of the monkey to appetitive and aversive signals. Electroencephogr Clin Neurophysiol 30:281-293.

Gaffan D, Harrison S (1989) Place memory and scene memory: effects of fornix transection in the monkey. Exp Brain Res 74:202-212.
Gardner-Medwin AR (1976) The recall of events through the learning of associations between their parts. Proc R Soc Lond [Biol] 194:375402.

Gross CG, Rocha-Miranda CF, Bender DB (1972) Visual properties of neurons in inferotemporal cortex of the macaque. $J$ Neurophysiol 35:96-111.

Gross CG, Bender DB, Gerstein GL (1979) Activity of inferior temporal neurons in behaving monkeys. Neuropsychologia 17:215-229.

Heit G, Smith ME, Halgren E (1988) Neural encoding of individual words and faces by the human hippocampus and amygdala. Nature 333:773-775.

Heit G, Smith ME, Halgren E (1990) Neuronal activity in the human medial temporal lobe during recognition memory. Brain 113:10931112.

Horel JA (1978) The neuroanatomy of amnesia. A critique of the hippocampal memory hypothesis. Brain 101:403-445.

Horel JA, Pytko-Joiner DE, Voytko M, Salsbury K (1987) The performance of visual tasks while segments of the inferotemporal cortex are suppressed by cold. Behav Brain Res 23:29-42.

Horn $G$ (1969) A simple device for making tungsten microelectrodes in bulk. J Physiol (Lond) 204:6P-7P.

Humphrey NK, Weiskrantz L (1969) Size constancy in monkeys with inferotemporal lesions. Q J Exp Psychol 21:225-238.

Ito M (1989) Long-term depression. Annu Rev Neurosci 12:85-102.

Jones EG, Powell TPS (1970) An anatomical study of converging sensory pathways within the cerebral cortex of the monkey. Brain 93 : 793-820.

Kandel ER (1981) Calcium and the control of synaptic strength by learning. Nature 293:697-700.

Konorski J (1959) A new method of physiological investigation of recent memory in animals. Bull Acad Pol Sci [Biol] 7:115-117.

Lorente de Nó R (1933) Studies on the structure of the cerebral cortex. I. The area entorhinalis. J Psychol Neurol (Leipzig) 45:381-438.

Lorente de Nó R (1934) Studies on the structure of the cerebral cortex. II. Continuation of the study of the ammonic system. J Psychol Neurol (Leipzig) 46:113-177.

Mahut H, Zola-Morgan S, Moss M (1982) Hippocampal resections impair associative learning and recognition memory in the monkey. J Neurosci 2:1214-1229.

Malamut BL, Saunders RC, Mishkin M (1984) Monkeys with combined amygdalo-hippocampal lesions succeed in object discrimination learning despite 24-hour intertrial intervals. Behav Neurosci 98 : 759-769.

Marr D (1971) Simple memory: a theory for archicortex. Philos Trans $R$ Soc Lond [Biol] 262:23-81.

Mayes AR (1988) Human organic memory disorders. Cambridge: Cambridge UP.

Mays LE, Best PJ (1975) Hippocampal unit activity to tonal stimuli during arousal from sleep and in awake rats. Exp Neurol 46:113-177.

Mikami A, Kubota B (1980) Inferotemporal neuron activities and color discrimination with delay. Brain Res 182:65-78.

Milner B (1970) Memory and the medial temporal regions of the brain. In: Biology of memory (Pribram KH, Broadbent DE, eds), pp 29-50. New York: Academic.

Mishkin M (1978) Memory in monkeys impaired by combined but not by separate removal of amygdala and hippocampus. Nature 273: 297-298.

Mishkin M (1982) A memory system in the monkey. Philos Trans R Soc Lond [Biol] 298:85-95.

Miyashita $Y$ (1988) Neuronal correlate of visual associative long-term memory in the primate temporal cortex. Nature 335:817-820.

Miyashita Y, Rolls ET, Cahusac PMB, Niki H, Feigenbaum JD (1989) Activity of hippocampal formation ncurons in the monkey related to a conditional spatial response task. J Neurophysiol 61:669-677.

Murray EA, Bachevalier J, Mishkin M (1989) Effects of rhinal cortical lesions on visual recognition memory in rhesus monkeys. Soc Neurosci Abstr 15:342.

O'Keefe J, Nadel L (1978) The hippocampus as a cognitive map. Oxford: Oxford UP.

Parkin AJ (1987) Memory and amnesia: an introduction. Oxford: Blackwell.

Richmond BJ, Sato T (1987) Enhancement of inferior temporal neurons during visual discrimination. J Neurophysiol 58:1292-1306.

Ringo JL, Doty RW (1985) A macaque remembers pictures briefly viewed six months earlier. Behav Brain Res 18:289-294. 
Rolls ET, Perrett DI, Caan AW, Wilson FAW (1982) Neuronal responses related to visual recognition. Brain 105:611-646.

Rolls ET, Miyashita Y, Cahusac PMB, Kesner RP, Niki H, Feigenbaum JD, Bach L (1989) Hippocampal neurons in the monkey with activity related to the place in which a stimulus is shown. J Neurosci 9:1835-1845.

Rosene DL, Van Hoesen GW (1977) Hippocampal cffcrents reach widespread areas of cerebral cortex and amygdala in the rhesus monkey. Science 198:315-317.

Rosene DL, Van Hoesen GW (1987) The hippocampal formation of the primate brain. A review of some comparative aspects of cytoarchitecture and connections. In: Cerebral cortex, Vol 6 (Jones EG, Peters A, eds), pp 345-456. New York: Plenum.

Russchen FT, Amaral DG, Price JL (1985) The afferent connections of the substantia innominata in the monkey, Macaca fascicularis. J Comp Neurol 242:1-27.

Schacter DL (1987) Implicit memory: history and current status. J Exp Psychol [Hum Learn] 13:501-518.

Scoville WB, Milner B (1957) Loss of recent memory after bilateral hippocampal lesions. J Neurol Neurosurg Psychiatry 20:11-21.

Seltzer B, Pandya DN (1978) Afferent cortical connections and architectonics of the superior temporal sulcus and surrounding cortex in the rhesus monkey. Brain Res 149:1-24.

Shimamura AP (1986) Priming effects in amnesia: evidence for a dissociable memory function. Q J Exp Psychol 38A:619-644.

Sidman M, Stoddard LT, Mohr JP (1968) Some additional quantitative observations of immediate memory in a patient with bilateral hippocampal lesions. Neuropsychologia 6:245-254.

Squire LR (1987) Memory and brain. New York: Oxford UP.

Squire LR, Zola-Morgan S, Chen KS (1988) Human amnesia and animal models of amnesia: performance of amnesic patients on tests designed for the monkey. Behav Neurosci 102:210-221.

Stepien LS, Cordeau JP, Rasmussen T (1960) The effect of temporal lobe and hippocampal lesions on auditory and visual recent memory in monkeys. Brain 83:470-489.

Thompson RF, Spencer WA (1966) Habituation: a model phenomenon for the study of neuronal substrates of behaviour. Psychol Rev 73:16-43.

Ungerleider LG, Ganz L, Pribram KH (1977) Size constancy in rhesus monkeys: effects of pulvinar, prestriate and inferotemporal lesions. Exp Brain Res 27:251-269.

Ungerleider LG, Gaffan D, Pelak VS (1989) Projections from inferior temporal cortex to prefrontal cortex via the uncinate fascicle in rhesus monkeys. Exp Brain Res 76:473-484.

Van Hoesen GW (1982) The parahippocampal gyrus. New observations regarding its cortical connections in the monkey. Trends Neurosci 5:345-350.

Van Hoesen GW, Pandya DN (1975) Some connections of the en- torhinal (area 28) and perirhinal (area 35) cortices of the rhesus monkey. 1. Temporal lobe afferents. Brain Res 95:1-24.

Van Hoesen GW, Yeterian EH, Lavizzo-Mourey R (1981) Widespread corticostriate projections from temporal cortex of the rhesus monkey. J Comp Neurol 199:205-219.

Vinogradova OS (1975) Functional organisation of the limbic system in the process of registration of information: facts and hypotheses. In: The hippocampus, Vol 2, Neurophysiclngy and behaviour (Isaacson RL, Pribram KH, eds), pp 3-67. Is is in: Plenum.

von Bonin G, Bailey P (1947) The nee e. of Macaca mulatta. Urbana: University of Illinois.

Watanabe T, Niki H (1985) Hippocampal unit activity and delayed response in the monkey. Brain Res 325:241-254.

Wilson FAW, Rolls ET (1987) Habituation and stimulus-specific neuronal responses recorded from the amygdala of the monkey. Soc Neurosci Abstr 13:305.6.

Wilson FAW, Rolls ET (1990a) Neuronal responses related to the novelty and familiarity of visual stimuli in the substantia innominata, diagonal band of Broca and the periventricular region of the primate basal forebrain. Exp Brain Res 80:104-120.

Wilson FAW, Rolls ET (1990b) Neuronal responses related to reinforcement in the primate basal forebrain. Brain Res 509:213-231.

Wilson FAW, Brown MW, Riches IP (1988) Neuronal activity in the inferomedial temporal cortex compared with that in the hippocampal formation. Implications for amnesia of medial temporal lobe origin. In: Cellular mechanisms of conditioning and behavioral plasticity (Woody CD, Alkon DL, McGaugh JL, eds), pp 313-328. New York: Plenum.

Wilson FAW, Riches IP, Brown MW (1990) Hippocampus and medial temporal cortex: neuronal activity related to behavioural responses during performance of memory tasks by primates. Behav Brain Res 40:7-28.

Witter MP, Van Hoesen GW, Amaral DG (1989) Topographical organisation of the entorhinal projection to the dentate gyrus of the monkcy. J Ncurosci 9:216-228.

Yeterian EH, Pandya DN (1988) Corticothalamic connections of paralimbic regions in the rhesus monkey. J Comp Neurol 269:130-146.

Zola-Morgan S, Squire LR (1985) Medial temporal lesions in monkeys impair memory on a variety of tasks sensitive to human amnesia. Behav Neurosci 99:22-34.

Zola-Morgan S, Squire LR, Amaral DG (1986) Human amnesia and the medial temporal region: enduring memory impairment following a bilateral lesion limited to field CAl of the hippocampus. J Neurosci 6:2950-2967.

Zola-Morgan S, Squire LR, Amaral DG, Suzuki WA (1989) Lesions of perirhinal and parahippocampal cortex that spare the amygdala and hippocampal formation produce severe memory impairment. $J$ Neurosci 9:4355-4370. 